\title{
Fermionic Molecular Dynamics
}

\author{
H. Feldmeier* and J. Schnack ${ }^{\dagger}$ \\ Gesellschaft für Schwerionenforschung mbH, \\ Postfach 110 552, D-64220 Darmstadt
}

\begin{abstract}
A quantum molecular model for fermions is investigated which works with antisymmetrized many-body states composed of localized single-particle wave packets. The application to the description of atomic nuclei and collisions between them shows that the model is capable to address a rich variety of observed phenomena. Among them are shell effects, cluster structure and intrinsic deformation in ground states of nuclei as well as fusion, incomplete fusion, dissipative binary collisions and multifragmentation in reactions depending on impact parameter and beam energy. Thermodynamic properties studied with long time simulations proof that the model obeys Fermi-Dirac statistics and time averaging is equivalent to ensemble averaging. A first order liquid-gas phase transition is observed at a boiling temperature of $T \approx 5 \mathrm{MeV}$ for finite nuclei of mass $16 \ldots 40$.
\end{abstract}

PACS: 24.10.Cn, 25.70.-z, 02.70.Ns, 05.30.-d, 05.30.Fk, 05.60.+w, 05.70.Fh

Keywords: Fermion system; Molecular dynamics; Fermionic Molecular Dynamics; Heavy ion collisions; Deeply inelastic reactions; Multifragmentation; Thermodynamic properties; Liquid-gas phase transition

*email: h.feldmeier@gsi.de, WWW: http://www.gsi.de/ feldm

$\dagger$ †email: j.schnack@gsi.de, WWW: http://www.gsi.de/ schnack 


\section{Contents}

$\begin{array}{lll}1 & \text { Introduction and summary } & 3\end{array}$

2 The concept of Fermionic Molecular Dynamics $\quad 6$

2.1 Parameterized many-body trial state . . . . . . . . . . . . . . . . . 9

2.2 Time-dependent variational principle . . . . . . . . . . . . . . . . 14

2.3 Conservation laws . . . . . . . . . . . . . . . . . 16

2.4 Two-body Hamiltonian . . . . . . . . . . . . . . . . . . . 18

2.4 .1 Effective nucleon-nucleon interaction . . . . . . . . . . . . 18

2.4 .2 Coulomb interaction . . . . . . . . . . . . . . . . . 19

3 Ground states in FMD 20

3.1 Ground states of nuclei . . . . . . . . . . . . . . . . . . . . . . . 21

3.2 Shell structure in FMD . . . . . . . . . . . . . . . . . . . . . . . . 23

$\begin{array}{lll}4 \text { Heavy ion reactions } & 25\end{array}$

4.1 Deeply inelastic reactions . . . . . . . . . . . . . . . . . . . 27

4.2 Multifragmentation . . . . . . . . . . . . . . . . . . . 30

5 Statistical properties of FMD 36

5.1 Thermostatic properties . . . . . . . . . . . . . . . . . . 38

5.1 .1 Completeness relation with coherent states . . . . . . . . . . 38

5.1 .2 The partition function . . . . . . . . . . . . . . . . . 40

5.1 .3 Example . . . . . . . . . . . . . . . . . . . 41

5.2 Canonical versus ergodic ensemble . . . . . . . . . . . . . . . 41

5.2.1 Canonical ensemble of fermions in a harmonic oscillator . . . . 43

5.2 .2 Ergodic ensemble of fermions in a harmonic oscillator . . . . . 44

5.2.3 Canonical and ergodic ensemble for distinguishable particles . 48

5.3 Caloric curve for finite nuclei . . . . . . . . . . . . . . . . . . . . . . . 49

\begin{tabular}{|ll}
\hline A Interaction matrix elements & 54
\end{tabular}

A.1 Approximation of the matrix elements . . . . . . . . . . . . . 54

A.2 Coulomb interaction . . . . . . . . . . . . . . . . 55 


\section{Introduction and summary}

Heavy-ion reactions show typical dissipative phenomena at low beam energies (a few $\mathrm{MeV}$ per nucleon above the Coulomb barrier) [1, 2]. With increasing impact parameter the complete fusion reactions go over into deeply inelastic collisions where the scattered nuclei have lost large fractions of the initial energy and converted that into intrinsic excitation energy. The exchange of nucleons causes the mass and charge numbers of the outgoing nuclei to fluctuate around a mean value. At the same time energy loss and scattering angle show fluctuations like a Brownian movement. For the description of these phenomena the so called particle exchange picture [3, 4, 5] which is based on the assumption of a Fermi gas with long mean free paths turned out to be rather successful.

Around the same time the time-dependent Hartree-Fock model (TDHF) [6, 7, 8, 9] was conceptually and numerically developed to a stage where heavy-ion collisions could be calculated. Although TDHF is a microscopic quantal model, which is supposed to describe systems with long mean free path and slow collective motion of the mean field, slow compared to the Fermi velocity such that the particles and their mean field can always be in equilibrium, it turned out that only the dissipation of the collective energy could be described but not the fluctuations [8] which inevitably go along with any dissipation. TDHF results are very close to a classical trajectory picture with friction and very small fluctuations.

The surprising failure of the more quantal and more microscopic TDHF model compared to the more phenomenological particle exchange picture, both being based mainly on independent particle motion, originates in the inability of the TDHF state to react to small fluctuations. Consider a symmetric system: if there is a fluctuation in one direction, let us say a nucleon passes from the left to the right hand nucleus, there is of course in a many-body quantum state, always with the same probability the fluctuation in the opposite direction. Thus the mean field which averages over all configurations, will not adjust to the new situation, namely that there is now one more particle in the right hand nucleus, because with exactly the same probability a particle went out to the other nucleus and thus the mean particle number stays the same. The same holds true for recoil effects which would give a fluctuation to the collective relative momentum. The time evolution with the Hartree-Fock 
Hamiltonian conserves global symmetries of the initial state. The mean field in TDHF is the same for all macro-channels (mass numbers, charge numbers, relative momenta etc.) which causes a "spurious cross channel coupling" 10 suppressing almost all fluctuations.

In the particle exchange picture a random transfer of a nucleon will cause a jump to a new mean field situation where the acceptor nucleus is now enlarged in volume such that the new radius is that of a nucleus with one more particle. This way the decision is made, which of the many possibilities in the many-body quantum state is realized and this new situation is then evolved further in time. Of course with the same probability the particle could have jumped to the other side, then this would be the new situation with a larger mean field on the other side which would be evolved further in time. This picture is much more realistic for dissipative phenomena because the exact solution of the Schrödinger equation develops random phases between the macro-channels such that they cannot interfere on a macroscopic scale.

When the beam velocity becomes comparable to the Fermi velocity the mean field picture of binary collisions should break down. In addition the mean free path of the nucleons becomes shorter than the diameter of the system due to the larger intrinsic excitation energy. In BUU-type models [11, 12, 13, 14, 15, 16] a random Boltzmann collision term has been introduced to account for the shorter mean free path, but the long range part of the two-body interaction is still treated in a mean field fashion. The next step has been to replace the mean-field part by classical molecular dynamics with soft phenomenological two-body potentials [17, 18]. These Quantum Molecular Dynamics (QMD) approaches have two main conceptional difficulties. First, the mean field (long range part of the interaction) and the collision term (short range repulsion) should be treated self-consistently [19, 20]. Second, the Pauli principle is reduced to the numerically enforced prohibition of over-occupation of phase-space cells. Other approaches try to replace the Pauli principle by adding a two-body "Pauli potential" in order to avoid too large onebody phase-space densities [21, 22, 23, 24, 25, 26]. The difficulty is that the Pauli principle requires the many-body state to be antisymmetric with respect to particle exchange, and this is outside the classical notions.

In the following sections we discuss a model, named Fermionic Molecular Dy- 
namics (FMD) [27, 28, 29], which combines the microscopic quantal features including the Pauli principle with the properties of classical molecular dynamics. Wave packets replace the classical points in phase space and thus introduce quantum properties into classical molecular dynamics. Especially the antisymmetrization of the many-body state has many consequences genuine in quantum and absent in classical mechanics.

A classical system of particles (molecules) which interact via a two-body interaction of van der Waals type - repulsive at short and attractive at large distance - often behaves in a deterministic chaotic way. Small deviations in the initial conditions lead to exponentially diverging trajectories in the many-body phase space. Situations with global symmetries, for instance the left-right symmetry in the TDHF solution discussed above, are of measure zero in the classical case. Each particle follows a single trajectory which cannot split into two or more with certain amplitudes like in the quantum case. This property is also common to FMD since there is only one wave packet per nucleon for which the equations of motion decide in a deterministic way where to move, for example whether it will join the right or left hand nucleus. This "quantization" of the particle number density is the main difference to TDHF if a single Slater determinant is used. In a forthcoming publication we are introducing antisymmetric many-body states which are not single Slater determinants anymore, but take care of the short range correlations caused by the repulsive core in the interaction.

After discussing the concept of the model and the resulting properties we calculate ground states of nuclei. In section 3 an important insight is that the antisymmetrization not only takes care of the Pauli principle, but also delocalizes the wave packets and introduces shell effects. The ground states turn out to be rather similar to deformed Hartree-Fock states.

The dynamical properties of the FMD equations of motion are investigated in section 1 . Calculations of heavy-ion collisions at $6 \mathrm{AMeV}$ show that the typical fluctuations of dissipative collisions, which are absent in TDHF, are seen in FMD. Increasing the beam energy to $32 \mathrm{AMeV}$ which corresponds to a relative velocity of about the Fermi velocity leads to a break down of the mean field. The very same model which describes fusion and binary dissipative collisions now predicts multifragmentation. 
A microscopic transport model developed for non-equilibrium situations should of course also possess the correct equilibrium properties. In the last section we proof that the thermostatic properties calculated with the FMD trial state are those of Fermi-Dirac statistics. Time averaging over dynamical calculations defining the thermodynamic properties not only shows that FMD equilibrates towards the Fermi distribution, but even allows to investigate the liquid-gas phase transition of small nuclei.

\section{The concept of Fermionic Molecular Dynamics}

The concept of Fermionic Molecular Dynamics is based on the molecular dynamics picture in which subgroups of the investigated system are described by their centre of mass coordinates. These subgroups are composite bound objects like molecules or in our case nucleons. The molecules are interacting by two-body potentials which approximate the complicated interactions between the constituents of the molecules. The two-body potentials, which include to a certain degree also the polarization induced in one molecule by the presence of the other one, depend in general on the relative distance, the relative velocity and the relative orientation of the molecules.

Consider for example the interaction $V_{w w}$ between water molecules which have a dipole moment. First, it is a function of the distance but also of the relative orientation (see fig. 1). Second, depending on the relative velocity the electron clouds will be able to adjust more or less completely to the adiabatic situation. This will result in a velocity dependence.

In our case the complex molecule is the nucleon which consists of three quarks surrounded by a cloud of virtual mesons. Like the water molecule the nucleon is not spherically symmetric but has an orientation, the spin. In addition there are two kinds of molecules, protons and neutrons, which introduces a further degree of freedom, the isospin. Altogether the picture of a nucleon-nucleon potential in itself is only an approximation and there is a certain degree of freedom in its choice.

In classical molecular dynamics the molecules are treated as classical distinguishable particles which means that one has to solve Newton's equations of motion for the classical centre of mass coordinates. The many-body state is given by the tra- 


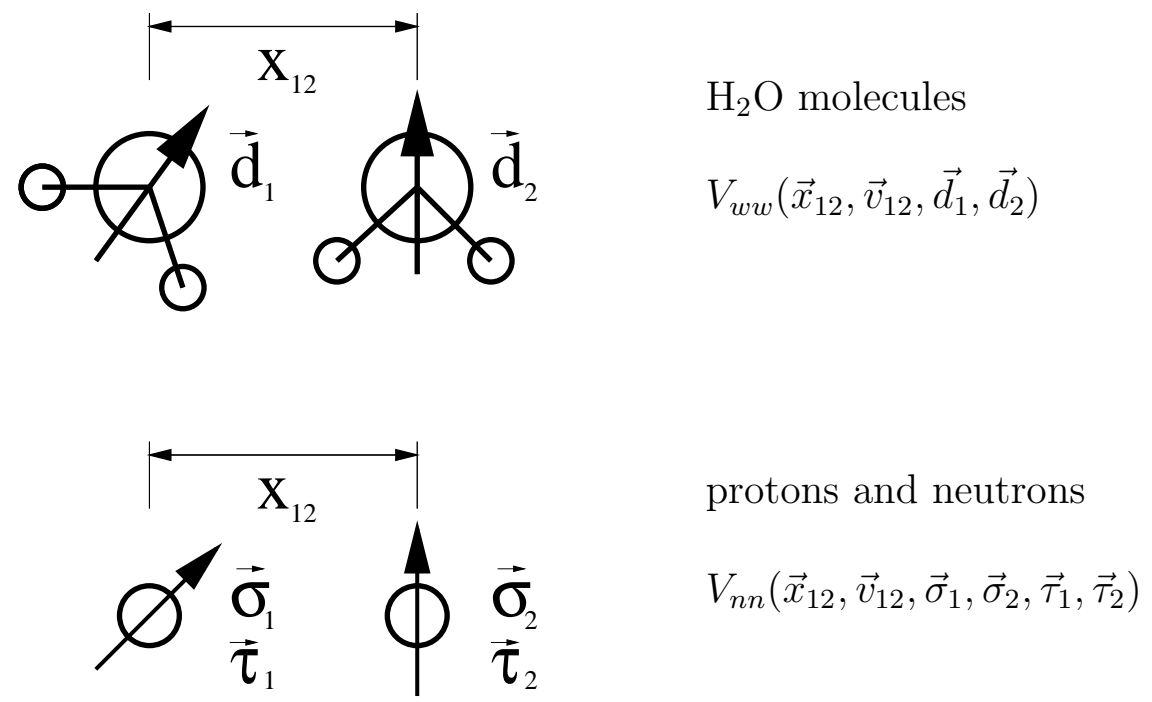

Figure 1: Interactions between water molecules and nucleons (protons or neutrons) are rather complex.

jectory in many-body phase space.

Here Fermionic Molecular Dynamics differs substantially because its many-body state is described by an antisymmetrized many-body wave function for the centre of mass coordinates and the spin degrees of freedom of all nucleons. As already explained in the introduction, in nuclei the nucleons cannot be localized in phase space well enough for a non-quantal treatment. The same holds true for the spin variables. Unlike the water molecule, where the dipole moment has only small quantum fluctuations around its mean value, the Hilbert space for the spin is only two-dimensional and hence one cannot construct a localized wave-packet for the spin orientation. A spin $\frac{1}{2}$ degree of freedom is always quantal.

All this together does not allow to treat nucleons in nuclei as classical particles. Therefore FMD is using localized single-particle wave packets for the centre of mass coordinates instead of points in phase space. The indistinguishability of the particles, which has such prominent consequences like Fermi motion, Fermi pressure, or Pauli blocking, is introduced by using a many-body state which is antisymmetric with respect to particle exchange. Therefore, all effects which arise from the fact that the particles are indistinguishable fermions are taken into account and by construction the Pauli principle cannot be violated. The use of wave packets guarantees that the uncertainty relation cannot be violated either. It actually turns out that due 
to antisymmetrization the localization of the particles is suspended when the wave packets are overlapping. Thus, shell model states as well as plane waves are included in $|Q\rangle$ even if it is built from Gaussian shaped single-particle states (see section 3.2)

This means that the trial state contains the essential quantum properties of a Fermi gas. In addition if the system is dilute and the distance between the particles is much larger than the widths of their wave packets one obtains the classical limit in the sense of Ehrenfest. Hence the FMD trial state has enough freedom to cover large areas of quantum physics and connects seamlessly to classical physics.

So the first part of the FMD concept is to include all necessary physics in the many-body trial state $|Q\rangle$ but keeping this many-body state still numerically treatable.

The second part of the concept is to deduce the equations of motion from the time-dependent quantum variational principle

$$
\delta \int_{t_{1}}^{t_{2}} \mathrm{~d} t\left\langle Q(t)\left|i \frac{d}{d t}-\underset{\sim}{H}\right| Q(t)\right\rangle=0 .
$$

This variational principle [30], which will be explained in more detail below, ensures automatically the conservation laws, provided the trial state has enough freedom as shown in section 2.3 .

Altogether, the concept is well defined and the success of the model is granted if one has the proper Hamiltonian $\underset{\sim}{\sim}$ and a rich enough trial state. The art consists in finding a trial state which contains the essential degrees of freedom without becoming numerically intractable. As one does not know a priori what the important physical phenomena will be, one needs experimental guidance and physical intuition to set up the trial state. Even for the same Hamiltonian $\underset{\sim}{H}$ there can be different optimal sets of trial states, depending on the excitation energy or the part of the phase space the system is occupying.

If the model fails, the concept is to reexamine the trial state and to include more or other degrees of freedom. But also the Hamiltonian is not sacred as it is in many cases only an approximation to a much more complex underlying microscopic picture.

There is also an interplay between the trial state and the effective Hamiltonian. 
In nuclear physics the nucleon-nucleon interaction is strongly repulsive at short distances. This important feature contributes to nuclear saturation and hence is part of the essential physics which should also be present in the trial state. The antisymmetrized product state (single Slater determinant) which we use in this paper cannot account for the depletion of the many-body wave function at small relative distances between the particles. Therefore, we are using an effective interaction with a moderate repulsion at short distances in the spirit of a Brueckner G-Matrix treatment.

\subsection{Parameterized many-body trial state}

Instead of classical points in phase space Fermionic Molecular Dynamics deals with wave packets which are localized in phase space. Each nucleon (molecule) is represented by a Gaussian wave packet parameterized by the set

$$
\begin{aligned}
q(t)= & \{\vec{r}(t), \vec{p}(t), a(t), \chi(t), \phi(t), \xi\}: \\
& \langle\vec{x} \mid q(t)\rangle=\exp \left\{-\frac{(\vec{x}-\vec{r}(t))^{2}}{2 a(t)}+i \vec{p}(t) \cdot \vec{x}\right\} \otimes|\chi(t), \phi(t)\rangle \otimes|\xi\rangle,
\end{aligned}
$$

which in quantum mechanics is the closest analogue to a classical particle described by a single point $(\vec{r}(t), \vec{p}(t))$ in phase-space.

In FMD the match to classical positions and momenta are the parameters $\vec{r}(t)$ and $\vec{p}(t)$ which determine the mean values of the position and momentum operator of the single particle state:

$$
\vec{r}(t)=\frac{\langle q(t)|\underset{\sim}{\vec{x}}| q(t)\rangle}{\langle q(t) \mid q(t)\rangle}, \vec{p}(t)=\frac{\langle q(t)|\underset{\sim}{\vec{k}}| q(t)\rangle}{\langle q(t) \mid q(t)\rangle} .
$$

Due to the quantum mechanical uncertainty relation the wave packet can be either narrow in coordinate space and wide in momentum space or vice versa. This nonclassical degree of freedom is taken care of by the complex width parameter $a(t)=$ $a_{R}(t)+i a_{I}(t)$. It determines via its real part $a_{R}(t)$ the variance of the momentum distribution $\sigma_{K}^{2}(t)$ by the relation

$$
\frac{3}{2 a_{R}(t)}=\frac{\left\langle q(t)\left|(\underset{\sim}{\vec{k}}-\vec{p}(t))^{2}\right| q(t)\right\rangle}{\langle q(t) \mid q(t)\rangle}=3 \sigma_{K}^{2}(t) .
$$


Since the wave packet is spherical the widths are equal in all three Cartesian directions. The imaginary part $a_{I}(t)$ appears in the expression for the spatial width $\sigma_{X}^{2}(t)$ as

$$
\frac{3}{2} \frac{a_{R}^{2}(t)+a_{I}^{2}(t)}{a_{R}(t)}=\frac{\left\langle q(t)\left|(\underset{\sim}{\vec{x}}-\vec{r}(t))^{2}\right| q(t)\right\rangle}{\langle q(t) \mid q(t)\rangle}=3 \sigma_{X}^{2}(t)
$$

and determines in how far the wave packet is of minimal uncertainty. The product of the variances

$$
\sigma_{X}^{2} \sigma_{K}^{2}=\frac{1}{4}\left(1+\frac{a_{I}^{2}}{a_{R}^{2}}\right)
$$

shows that for $a_{I}=0$ one has the minimum-uncertainty packet where $\sigma_{X} \sigma_{K}=\frac{1}{2}$, while for $a_{I} \neq 0$ the uncertainty can become arbitrarily large. This means that the particle occupies more than $(\hbar / 2 \pi)^{3}$ of phase space volume but at a lower phase space density, such that other fermions can find place at the same area in phase space.

Besides the parameters for the spatial part of the wave packet there are two parameters $\chi(t)$ and $\phi(t)$ for the spin degree of freedom. If one parameterizes the trial spin-state by

$$
\left\langle m_{s} \mid \chi(t), \phi(t)\right\rangle=\left\{\begin{array}{ll}
\cos \frac{\chi(t)}{2} & : m_{s}=\frac{1}{2} \\
\sin \frac{\chi(t)}{2} \mathrm{e}^{i \phi(t)} & : m_{s}=-\frac{1}{2}
\end{array},\right.
$$

the relation between the parameters and the corresponding spin operators is

$$
\vec{\sigma}(t)=\frac{\langle q(t)|\underset{\sim}{\vec{\sigma}}| q(t)\rangle}{\langle q(t) \mid q(t)\rangle}
$$

where $\vec{\sigma}(t)=(\sin \chi(t) \cos \phi(t), \sin \chi(t) \sin \phi(t), \cos \chi(t))$ is a vector in the 3 -dimensional real space and the quantization axis is the 3 -axis. Thus $\vec{\sigma}(t)$ can be regarded as the "classical" spin direction just like $\vec{r}(t)$ or $\vec{p}(t)$, although there is no classical spin degree of freedom which can vary only its direction but not its magnitude as it is the case for $\vec{\sigma}(t)$.

In principle the same parameterization can be chosen for the isospin part $|\xi\rangle$. A time-dependent isospin would mean that for example due to the exchange of charged pions neutrons can dynamically transform into protons and vice versa. Isospin symmetry is, however, only an approximate symmetry, the Coulomb interaction and the 
difference in proton and neutron mass break it. A linear superposition of protons and neutrons in one wave packet would impose undesired artificial symmetries. For example only the proton component would feel the Coulomb repulsion and its centre of the wave packet would be accelerated away from the centre for the neutron component. Therefore, either one gives each component its own wave packet or one does not allow proton neutron mixing in the trial state. In this paper we shall not consider rotations in isospin space but assume $|\xi\rangle$ to be independent of time and either $|\xi\rangle=\mid$ proton $\rangle$ or $|\xi\rangle=\mid$ neutron $\rangle$.

To describe a system with $A$ fermions we construct a Slater determinant $|Q(t)\rangle$ with these parameterized single-particle states $\left|q_{k}(t)\right\rangle$

$$
\left|q_{1}(t), q_{2}(t), \cdots, q_{A}(t)\right\rangle \equiv|Q(t)\rangle=\frac{1}{\langle\widehat{Q(t)} \mid \widehat{Q(t)}\rangle^{\frac{1}{2}}}|\widehat{Q(t)}\rangle,
$$

where the antisymmetrized but not normalized state $|\widehat{Q(t)}\rangle$ is given by

$$
|\widehat{Q(t)}\rangle=\frac{1}{A !} \sum_{\text {all } \pi} \operatorname{sgn}(\pi)\left|q_{\pi(1)}(t)\right\rangle \otimes\left|q_{\pi(2)}(t)\right\rangle \otimes \cdots \otimes\left|q_{\pi(A)}(t)\right\rangle .
$$

The sum runs over all permutations $\pi$ and $\operatorname{sgn}(\pi)$ is the sign of the permutation. It should be noted that $q_{k}(t)=\left\{\vec{r}_{k}(t), \vec{p}_{k}(t), a_{k}(t), \chi_{k}(t), \phi_{k}(t), \xi_{k}\right\}$ denotes the set of parameters specifying the single-particle state with number $k$. The parameter set for the many-body state thus reads

$$
\begin{aligned}
Q(t) & =\left\{\vec{r}_{1}(t), \vec{p}_{1}(t), a_{1}(t), \chi_{1}(t), \phi_{1}(t), \xi_{1} ; \vec{r}_{2}(t), \cdots ; \vec{r}_{A}(t), \cdots, \xi_{A}\right\} \\
& =\left\{q_{\nu}(t) \mid \nu=1, \cdots, \mathrm{N} A\right\}
\end{aligned}
$$

where $\mathrm{N}$ is the number of real parameters per particle, in our case $\mathrm{N}=10$. Whenever $q$ carries a Greek index it is an individual parameter, whereas a Latin index implies that $q_{k}$ is the whole set for the state $\left|q_{k}\right\rangle$.

Due to antisymmetrization FMD is constrained to the antisymmetric subspace of the Hilbert space and hence the Pauli principle is a priori incorporated. Furthermore, the projection (10) from a product state onto the antisymmetric subspace destroys for overlapping Gaussians the localization of the particles and introduces shell model states. This will be discussed and explicitly shown in section 3.2. If the single-particle states $\left|q_{k}\right\rangle$ are not overlapping, the antisymmetrization has no 
effect anymore and the particles are localized in the individual wave packets. In this limit we return to classical Newtonian mechanics for $\vec{r}_{k}(t)$ and $\vec{p}_{k}(t)$, which however can still be coupled to the non-classical variables $\vec{\sigma}_{k}(t)$ for the spin directions and the widths $a_{k}(t)$. Of course also in this limit the particles are indistinguishable and it is not possible to decide which particle occupies which Gaussian packet.

Even though eqs. (3) - (5) and (8) provide unique relations between the parameters and the expectation values of the corresponding operators, this is no longer true for the antisymmetrized many-body state. To illustrate this important aspect let us regard a two-body state of two packets which for simplicity have the same real width parameter $a_{1}=a_{2}=a_{R}$ and the same spin, but different $\vec{r}_{1}, \vec{r}_{2}$ and $\vec{p}_{1}, \vec{p}_{2}$

$$
\left|q_{1}, q_{2}\right\rangle_{a}=\frac{1}{\sqrt{2 N}}\left\{\left|q_{1}\right\rangle \otimes\left|q_{2}\right\rangle-\left|q_{2}\right\rangle \otimes\left|q_{1}\right\rangle\right\} .
$$

with the normalization

$$
\begin{aligned}
N & =\left\langle q_{1} \mid q_{1}\right\rangle\left\langle q_{2} \mid q_{2}\right\rangle-\left|\left\langle q_{1} \mid q_{2}\right\rangle\right|^{2} \\
& =\left\langle q_{1} \mid q_{1}\right\rangle\left\langle q_{2} \mid q_{2}\right\rangle\left(1-\exp \left\{-\xi_{12}^{2}\right\}\right), \quad \xi_{12}^{2}=\left(\vec{r}_{1}-\vec{r}_{2}\right)^{2} / a_{R}-\left(\vec{p}_{1}-\vec{p}_{2}\right)^{2} a_{R},
\end{aligned}
$$

where $\xi_{12}$ measures the distance in phase space.

The operator for the position of particle $1, \underset{\sim}{\sim}(1)=\underset{\sim}{\vec{x}} \otimes \underset{\sim}{\mathbb{1}}$, (which by the way is not an observable because it is not symmetric under particle permutation) has the following expectation value

$$
\begin{aligned}
& a\left\langle q_{1}, q_{2}|\underset{\sim}{\vec{x}}(1)| q_{1}, q_{2}\right\rangle_{a} \\
& =\frac{1}{2 N}\left\{\left\langle q_{1}|\underset{\sim}{\sim}| q_{1}\right\rangle+\left\langle q_{2}|\underset{\sim}{\vec{x}}| q_{2}\right\rangle-2 \operatorname{Re}\left(\left\langle q_{1}|\underset{\sim}{\vec{x}}| q_{2}\right\rangle\left\langle q_{2} \mid q_{1}\right\rangle\right)\right\} \\
& =\frac{1}{2}\left(\vec{r}_{1}+\vec{r}_{2}\right) .
\end{aligned}
$$

The result is not $\vec{r}_{1}$ as presumed from Ehrenfest type arguments, however, $\langle\underset{\sim}{\vec{x}}(1)+\underset{\sim}{\vec{x}}(2)\rangle=\vec{r}_{1}+\vec{r}_{2}$.

Another example is the relative distance between two identical fermions, $\underset{\sim}{\sim}(1)-$ $\underset{\sim}{\vec{x}}(2)$, (again not an observable, but used for example in its classical meaning in the collision term of QMD [18]) with the expectation value

$$
{ }_{a}\left\langle q_{1}, q_{2}|\underset{\sim}{\vec{x}}(1)-\underset{\sim}{\vec{x}}(2)| q_{1}, q_{2}\right\rangle_{a}=0
$$

If the spin of the two fermions is the same, the two-body state has to be antisymmetric in coordinate space and hence this expectation value is always zero, independent 
on the actual value of $\vec{r}_{1}$ and $\vec{r}_{2}$. The same holds true if the spins are anti-parallel. If the spins are not parallel the "distance" depends on the spin orientations, which only reflects that $\vec{r}_{1}-\vec{r}_{2}$ is not the mean distance between the two fermions.

An observable is for example the rms-distance $|\underset{\sim}{\sim}(1)-\underset{\sim}{\sim}(2)|$ which, however, also depends on the spin directions and the relative momentum. The reason is that for the component of the trial state in which the two fermions have total spin $S=0$ the radial part of the wave function is symmetric, while for the three $S=1$ components it is antisymmetric. Hence the rms-distance is different for the singlet and the triplet component. In addition the exchange term contributes less if the relative momentum becomes large. The Pauli principle, which demands that two fermions cannot be in the same phase space cell, is of course the origin of these classically not existing correlations in the rms-distance.

Another example for an observable which is even used to motivate a so called "Pauli potential" is the kinetic energy [23, 24]. For equal spins and equal widths $a_{1}=a_{2}=a_{R}$ we get

$$
\begin{aligned}
\langle\underset{\sim}{\sim}\rangle= & \frac{1}{2 m}{ }_{a}\left\langle q_{1}, q_{2}\left|\underset{\vec{k}}{\sim}(1)+\overrightarrow{\vec{k}}^{2}(2)\right| q_{1}, q_{2}\right\rangle_{a} \\
= & \frac{1}{2 m} \frac{1}{N}\left\{\left\langle q_{1}|\underset{\vec{k}}{\sim}| q_{1}\right\rangle+\left\langle q_{2}\left|\overrightarrow{\vec{k}}^{2}\right| q_{2}\right\rangle-2 \operatorname{Re}\left(\left\langle q_{1}|\underset{\sim}{\sim} \underset{\sim}{\sim}| q_{2}\right\rangle\left\langle q_{2} \mid q_{1}\right\rangle\right)\right\} \\
= & \frac{1}{2 m} \frac{\frac{1}{2}\left(\vec{p}_{1}-\vec{p}_{2}\right)^{2}+\left(\vec{r}_{1}-\vec{r}_{2}\right)^{2} / a_{R} \exp \left\{-\xi_{12}^{2}\right\}}{1-\exp \left\{-\xi_{12}^{2}\right\}} \\
& +\frac{1}{4 m}\left(\vec{p}_{1}+\vec{p}_{2}\right)^{2}+\frac{3}{2 m a_{R}} .
\end{aligned}
$$

One sees that the kinetic energy is not simply $\left(\vec{p}_{1}^{2}+\vec{p}_{2}^{2}\right) /(2 m)$ but depends also on the "relative distance" $\vec{r}_{1}-\vec{r}_{2}$ and on the distance in phase space $\xi_{12}$. But one has to be aware that for wave packets which are not of minimal uncertainty (i.e. the imaginary part $a_{I}$ of the width parameter $a$ is not zero) the whole expression is completely different and $\left|\left\langle q_{2} \mid q_{1}\right\rangle\right|^{2}$ can be zero even when the packets fully overlap spatially. A non-vanishing $a_{I}$ is nothing exceptional, it describes for example the well-known spreading of the packets in free space. Therefore, the constraint $a_{I}=0$ is not appropriate for the dynamical case and leads actually to unphysical scatterings and strong hindrance of evaporation.

In section 3.2 we shall show for an $A$-body system that even if all parameters for the momenta vanish, i.e. $\vec{p}_{1}=\vec{p}_{2}=\cdots=\vec{p}_{A}=0$, the kinetic energy is neither zero 
nor the sum of the zero-point energies $3 /\left(2 m a_{R}\right)$, but the momentum distribution is that of a Fermi gas with a sharp edge at the Fermi momentum.

To summarize this discussion, one should always carefully distinguish between parameters and physical observables. The parameters are in general not the expectation values of the corresponding operators.

\subsection{Time-dependent variational principle}

After having set up the many-body trial state $|Q(t)\rangle$ one has to construct equations of motion for the set of parameters $Q(t)=\left\{q_{\nu}(t) \mid \nu=1,2,3, \cdots\right\}$ which are the generalized coordinates of the system. As shown in the previous section the parameters may loose their original physical meaning due to antisymmetrization. Therefore one must not simply use the classical equations of motion for the position and momenta. In addition, even without antisymmetrization, classical physics does not tell how the complex width parameter $a_{k}$ should be evolved in time. Also the equations for the angles $\left(\chi_{k}, \phi_{k}\right)$ of the spin direction $\vec{\sigma}$, which turn out to be of the Bargmann-Michel-Telegdi type [31], are not self-evident.

Therefore, the equations of motion for the Fermionic Molecular Dynamics model are derived from the following time-dependent variational principle

$$
\delta \int_{t_{1}}^{t_{2}} d t\left\langle Q(t)\left|i \frac{d}{d t}-\underset{\sim}{H}\right| Q(t)\right\rangle=0
$$

in which the trial state $|Q(t)\rangle$ is to be varied. This has the advantage that the geometry of the manifold of trial states is automatically taken care of, irrespective of how the parameters of the states are defined. Furthermore, conservation laws follow in a transparent way from invariance properties of the trial state and the Hamiltonian, see section 2.3.

The variation has to be performed with respect to each parameter $q_{\nu}(t)$ with the end points kept fixed, i.e. $\delta q_{\nu}\left(t_{1}\right)=\delta q_{\nu}\left(t_{2}\right)=0$. The operator $\underset{\sim}{H}$ is the total Hamiltonian of the system. (Throughout the paper operators in Hilbert space will be underlined with a twiddle to distinguish them from parameters or expectation values.) 
The Euler-Lagrange equations

$$
\frac{d}{d t} \frac{\partial \mathcal{L}}{\partial \dot{q}_{\nu}}-\frac{\partial \mathcal{L}}{\partial q_{\nu}}=0 \quad, \quad \nu=1,2, \cdots, N
$$

which result from the variation (17) are written in terms of the Lagrange function

$$
\begin{aligned}
\mathcal{L}(Q(t), \dot{Q}(t)) & :=\left\langle Q(t)\left|i \frac{d}{d t}-\underset{\sim}{H}\right| Q(t)\right\rangle \\
& =\mathcal{L}_{0}(Q(t), \dot{Q}(t))-\mathcal{H}(Q(t))
\end{aligned}
$$

with

$$
\mathcal{L}_{0}(Q(t), \dot{Q}(t)):=\left\langle Q(t)\left|i \frac{d}{d t}\right| Q(t)\right\rangle=\sum_{\nu}\left\langle Q(t)\left|i \frac{\partial}{\partial q_{\nu}}\right| Q(t)\right\rangle \dot{q}_{\nu}
$$

in which $\dot{Q}(t)=\left\{\dot{q}_{\nu}(t) \equiv d q_{\nu} / d t \mid \nu=1,2, \cdots\right\}$ is the set of generalized velocities and $\mathcal{H}(Q(t))$ is the Hamilton function defined as the expectation value of the Hamiltonian $\underset{\sim}{H}:$

$$
\mathcal{H}(Q(t))=\langle Q(t)|\underset{\sim}{\sim}| Q(t)\rangle
$$

Different from classical mechanics the Lagrange function (19) is linear in the velocities $\dot{q}_{\nu}$ but at the same time the set $Q(t)=\left\{q_{\nu}(t)\right\}$ contains both, coordinates and momenta.

Using the general structure (19) of the Lagrange function the Euler-Lagrange equations (18) in their most general form can be written as

$$
\sum_{\nu} \mathcal{A}_{\mu \nu}(Q) \dot{q}_{\nu}=-\frac{\partial \mathcal{H}(Q)}{\partial q_{\mu}}
$$

or, if $\mathcal{A}_{\mu \nu}$ is not singular [32] the equations of motion are

$$
\dot{q}_{\mu}=-\sum_{\nu} \mathcal{A}_{\mu \nu}^{-1}(Q) \frac{\partial \mathcal{H}(Q)}{\partial q_{\nu}}
$$

where

$$
\mathcal{A}_{\mu \nu}(Q)=-\mathcal{A}_{\nu \mu}(Q)=\frac{\partial^{2} \mathcal{L}_{0}}{\partial \dot{q}_{\mu} \partial q_{\nu}}-\frac{\partial^{2} \mathcal{L}_{0}}{\partial \dot{q}_{\nu} \partial q_{\mu}}
$$

is a skew symmetric matrix, which depends in general on all variables $Q(t)=\left\{q_{\nu}(t)\right\}$. For details see ref. [28]. 
With help of the matrix $\mathcal{A}_{\mu \nu}$ one can define generalized Poisson brackets 30 as

$$
\{\mathcal{H}, \mathcal{B}\}:=\sum_{\mu, \nu} \frac{\partial \mathcal{H}}{\partial q_{\mu}} \mathcal{A}_{\mu \nu}^{-1} \frac{\partial \mathcal{B}}{\partial q_{\nu}}
$$

such that the time derivative of an expectation value

$$
\mathcal{B}(t)=\langle Q(t)|\stackrel{B}{\sim}| Q(t)\rangle
$$

of a time-independent operator $\underset{\sim}{B}$ calculated with the trial state $|Q(t)\rangle$ is given by

$$
\begin{aligned}
\frac{d}{d t} \mathcal{B}(t) & =\frac{d}{d t}\langle Q(t)|\underset{\sim}{B}| Q(t)\rangle=\sum_{\nu} \dot{q}_{\nu} \frac{\partial \mathcal{B}}{\partial q_{\nu}} \\
& =\sum_{\mu, \nu} \frac{\partial \mathcal{H}}{\partial q_{\mu}} \mathcal{A}_{\mu \nu}^{-1} \frac{\partial \mathcal{B}}{\partial q_{\nu}}=\{\mathcal{H}, \mathcal{B}\} .
\end{aligned}
$$

Equation (27) has the symplectic manifold structure of Hamiltonian dynamics [33, but in the general case the parameters $q_{\nu}$ cannot be grouped into pairs of canonical variables. However, according to Darboux's theorem [33] canonical variables exist locally. They are non-linear functions of the parameters $q_{\nu}$ and have to be constructed such that $\mathcal{A}_{\mu \nu}^{-1}$ assumes the canonical form

$$
\mathcal{A}_{\mu \nu}^{-1}=\left(\begin{array}{cc}
0 & -\mathbf{1} \\
\mathbb{1} & 0
\end{array}\right),
$$

where $\mathbb{1}$ is the unit matrix. Their choice, however, is not unique.

\subsection{Conservation laws}

After having solved the equations of motion (23) for the parameters $Q(t)=\left\{q_{\nu}(t) \mid \nu=\right.$ $1, \cdots, \mathrm{N}\}$ the trial state $|Q(t)\rangle$ is known at all times. Thus one can calculate the expectation value $\mathcal{G}(t)=\langle Q(t)|\underset{\sim}{\sim}| Q(t)\rangle$ of an arbitrary time-independent operator $\underset{\sim}{\sim}$. With the definition (25) for the Poisson brackets the time derivative of this expectation value can be written as

$$
\frac{d}{d t} \mathcal{G}=\{\mathcal{H}, \mathcal{G}\}
$$

The expectation value is conserved in time if [34]

$$
\{\mathcal{H}, \mathcal{G}\}=\sum_{\mu, \nu} \frac{\partial \mathcal{H}}{\partial q_{\mu}} \mathcal{A}_{\mu \nu}^{-1} \frac{\partial \mathcal{G}}{\partial q_{\nu}}=0
$$


Since $\mathcal{A}_{\mu \nu}^{-1}$ is skew symmetric the energy $\mathcal{H}$ itself is always conserved by the equations of motion, provided they are derived from the variational principle (17). This is completely independent on the choice of the trial state .

For other constants of motion we consider a unitary transformation with $\underset{\sim}{G}$ as the hermitean generator

$$
\underset{\sim}{U}=\exp (i \epsilon \underset{\sim}{G}) \quad, \quad \epsilon \text { real } .
$$

If $\underset{\sim}{U}$ maps the set of trial states onto itself

$$
\underset{\sim}{U}|Q\rangle=\left|Q^{\prime}\right\rangle \quad \in \quad\{|Q\rangle\}
$$

then, as a result of the equations of motion, it can be shown [28] that

$$
\{\mathcal{H}, \mathcal{G}\}=\langle Q(t)|i[\underset{\sim}{H}, \underset{\sim}{G}]| Q(t)\rangle
$$

This means that for this class of generators the generalized Poisson bracket is just the expectation value of the commutator with $\underset{\sim}{i}$.

Relation (33) is very useful for two reasons. First, if $\underset{\sim}{G}$ commutes with the Hamiltonian $\underset{\sim}{H}$ and $\exp (i \in \underset{\sim}{\sim})|Q\rangle=\left|Q^{\prime}\right\rangle$ then $\langle Q(t)|\underset{\sim}{G}| Q(t)\rangle$ is automatically a constant of motion.

Second, this relation is an important guidance for the choice of the trial state $|Q\rangle$. If one wants the model to obey certain conservation laws then the set of trial states should be invariant under the unitary transformations generated by the constants of motion. For example, total momentum conservation implies that a translated trial state is again a valid trial state. This is fulfilled for the trial states specified in section 2.1. The Gaussians defined in eq. (2) can be translated or Galilei boosted, the latter taking care of the conservation of the centre of gravity.

Conservation of total spin $\underset{\sim}{\vec{J}}=\underset{\sim}{\vec{\sim}}+\underset{\sim}{\vec{S}}$ is guaranteed if rotation of the trial state in coordinate and spin space results again in a trial state. This implies that the Gaussian (2) has to have either a spherical shape or the width parameter has to be replaced by a complete tensor with 12 real parameters. It also means that all spin directions in $|\phi, \chi\rangle$ have to be allowed, otherwise the rotation would in general lead out of the manifold of trial states.

If $\underset{\sim}{G}$ does not commute with $\underset{\sim}{H}$, relation (33) sheds some light on the quality of the variational principle (17). It says that under the premise that $\exp (i \in \underset{\sim}{\sim})$ does not 
map out of the set of trial states the time derivative of the expectation value of $\underset{\sim}{G}$ calculated with a trial state is exact.

$$
\frac{d}{d t} \mathcal{G}(t)=\frac{d}{d t} \mathcal{G}_{\text {exact }}(t)
$$

where the exact solution with the initial state $|Q(t)\rangle$ is

$$
\mathcal{G}_{\text {exact }}(t+\tau)=\left\langle Q(t)\left|\mathrm{e}^{i \tau \stackrel{H}{\sim}} \underset{\sim}{\sim} \mathrm{e}^{-i \tau \stackrel{H}{\sim}}\right| Q(t)\right\rangle
$$

Due to the fact that $|Q(t+\tau)\rangle$ is only the approximate time evolution of $|Q(t)\rangle$ for some time $\tau$ later $\mathcal{G}(t+\tau)$ will begin to deviate from $\mathcal{G}_{\text {exact }}(t+\tau)$ for larger $\tau$.

The kinetic energy $\underset{\sim}{T}$ is such a generator. Since our trial state (9) is the exact solution of the Schrödinger equation without interactions it fulfills

$$
\exp (-i \tau \underset{\sim}{T})|Q(t)\rangle=|Q(t+\tau)\rangle
$$

With the two-body interaction included, $|Q(t)\rangle$ is not an exact solution anymore, but the expectation value of the total kinetic energy, which then is not a conserved quantity any longer, is well approximated in the sense of equation (34).

\subsection{Two-body Hamiltonian}

\subsubsection{Effective nucleon-nucleon interaction}

Up to now the interaction $\underset{\sim}{V}$ contained in the Hamiltonian was not specified. Since in this paper we shall investigate small and medium-heavy nuclei, we choose an effective two-body potential suited for mass numbers up to about 50. This interaction is repulsive at small and attractive at larger distances. The repulsive core is, however, rather weak and one should regard the potential as a phenomenological ansatz for a $\mathrm{G}$-matrix rather than the free nucleon-nucleon interaction which has a very strong repulsion for distances smaller than $0.5 \mathrm{fm}$. In this paper we are using a potential of the form

$$
\begin{aligned}
& \underset{\sim}{V}(i, j)=\underset{\sim}{V}\left(w_{a}+m_{a} \stackrel{P}{\sim}^{r}+b_{a} \underset{\sim}{P^{\sigma}}+h_{a} \stackrel{P}{\sim}^{\tau}\right) \\
& +\underset{V_{b}}{\sim}\left(w_{b}+m_{b} \stackrel{P}{\sim}^{r}+b_{b} \stackrel{P}{\sim}^{\sigma}+h_{b} \stackrel{P}{\sim}^{\tau}\right) \\
& +\underset{\sim}{V_{c}}\left(w_{c}+m_{c} \stackrel{P}{\sim}^{r}+b_{c} \stackrel{P}{\sim}^{\sigma}+h_{c} P^{\tau}\right)
\end{aligned}
$$


where $\underset{\sim}{P^{r}},{\underset{\sim}{\sim}}^{\sigma},{\underset{\sim}{\sim}}^{\tau}$ denote the various exchange operators for coordinate, spin and isospin. The radial dependences are of Gaussian type given by

$$
\begin{aligned}
\left\langle\vec{x}_{i}, \vec{x}_{j}\left|{\underset{\sim}{a, b, c}}_{a}(i, j)\right| \vec{x}_{k}, \vec{x}_{l}\right\rangle= & V_{a, b, c} \exp \left\{-\frac{\left(\vec{x}_{i}-\vec{x}_{j}\right)^{2}}{r_{a, b, c}^{2}}\right\} \\
& \times \delta^{3}\left(\vec{x}_{i}-\vec{x}_{k}\right) \delta^{3}\left(\vec{x}_{j}-\vec{x}_{l}\right)
\end{aligned}
$$

which allows to calculate all matrix elements and their derivatives analytically. The parameters

$$
\begin{aligned}
& V_{a}=-7.10 \mathrm{MeV} ; \quad r_{a}=1.16 \mathrm{fm} \\
& V_{b}=-31.90 \mathrm{MeV} ; \quad r_{b}=2.22 \mathrm{fm} \\
& V_{c}=+81.65 \mathrm{MeV} ; \quad r_{c}=0.735 \mathrm{fm} \\
& w_{a}=8.700, \quad m_{a}=5.610, \quad b_{a}=7.860, \quad h_{a}=-21.170 \\
& w_{b}=0.133, \quad m_{b}=0.514, \quad b_{b}=0.085, \quad h_{b}=0.268 \\
& w_{c}=1.000, \quad m_{c}=0.000, \quad b_{c}=0.000, \quad h_{c}=0.000
\end{aligned}
$$

were determined in order to reproduce the binding energies of a wide range of medium-heavy isotopes.

\subsubsection{Coulomb interaction}

The Coulomb interaction is included in the Hamilton operator, it is given by

$$
\left\langle\vec{x}_{i}, \vec{x}_{j}\left|\underset{\sim}{V_{c}}(i, j)\right| \vec{x}_{k}, \vec{x}_{l}\right\rangle=\frac{1.44 \mathrm{MeVfm}}{\left|\vec{x}_{i}-\vec{x}_{j}\right|} \underset{\sim}{\sim} \otimes \underset{\sim}{\sim}{ }^{p} \delta^{3}\left(\vec{x}_{i}-\vec{x}_{k}\right) \delta^{3}\left(\vec{x}_{j}-\vec{x}_{l}\right),
$$

where $\underset{\sim}{\sim}$ denotes the projection operator on the protons

$$
\stackrel{P}{\sim}^{p}\left|q_{m}\right\rangle=\frac{1}{2}\left(1+\xi_{m}\right)\left|q_{m}\right\rangle
$$

The isospin variable $\xi_{m}$ takes values $\xi_{m}=1$ for protons and $\xi_{m}=-1$ for neutrons, respectively.

In order to speed up computing time the expectation values of the two-body potentials are approximated as given in the appendix and spin degrees have been kept fixed in the following calculations. 


\section{Ground states in FMD}

The ground state of a nucleus is the many-body state $\left|Q_{G S}\right\rangle$ in which the energy $\mathcal{H}=\left\langle Q_{G S}|\underset{\sim}{\sim}| Q_{G S}\right\rangle$ is an absolute minimum with respect to variation of all parameters $q_{\nu}$, therefore

$$
\frac{\partial}{\partial q_{\nu}} \mathcal{H}=0
$$

This implies that the FMD ground state is completely time-independent (up to an overall phase) and the time derivatives of all parameters vanish because, by definition, all generalized forces $\partial \mathcal{H} / \partial q_{\mu}$ are zero and hence

$$
\dot{q}_{\nu}=-\sum_{\mu} \mathcal{A}_{\nu \mu}^{-1} \frac{\partial \mathcal{H}}{\partial q_{\mu}}=0 .
$$

Requirement (42) not only determines the positions $\vec{r}_{k}$ and momenta $\vec{p}_{k}$ but also the complex widths $a_{k}$ and the spin directions $\left(\chi_{k}, \phi_{k}\right)$.

Since we do not correct for the centre of mass motion in the dynamical calculation we use the expectation value of the Hamilton operator as the ground state energy and do not subtract the centre of mass energy. The centre of mass energy is of the order of $10 \mathrm{MeV}$ for all isotopes, so that its contribution to the energy per particle vanishes for larger mass numbers. An ansatz for the many-body state where centre of mass and relative motion separate is introduced in ref. 335.

Besides the ground state energy we also calculate the root-mean-square radius of the charge distribution.

$$
\begin{aligned}
E_{G S} & =\left\langle Q_{G S}|\underset{\sim}{\sim}| Q_{G S}\right\rangle \\
R_{r m s}^{2} & =\frac{1}{Z} \sum_{i=1}^{A}\left\langle Q_{G S}\left|\left(\underset{\sim}{\vec{x}}(i)-{\underset{\sim}{X}}_{C M}\right)^{2} \stackrel{P}{\sim}^{p}(i)\right| Q_{G S}\right\rangle+R_{\text {proton }}^{2}
\end{aligned}
$$

where the centre of mass position operator is

$$
\underset{\vec{X}_{C M}}{\vec{X}}=\frac{1}{A} \sum_{i=1}^{A} \underset{\vec{x}}{\sim}(i) .
$$

The operator $\underset{\sim}{P^{p}}(i)$ projects on protons and $R_{\text {proton }}=0.876 \mathrm{fm}$ takes the finite charge radius of the proton into account. 


\subsection{Ground states of nuclei}

In table 1 we summarize ground state binding energies and charge radii and compare them with experimental results. The interaction (37) describes a wide range of isotopes with a satisfying accuracy.

\begin{tabular}{|c||c|c|c|c||c|c|}
\hline \multicolumn{1}{|c||}{} & \multicolumn{2}{c|}{ experiment } & \multicolumn{2}{c||}{ FMD } & experiment & FMD \\
Isotope & $\begin{array}{c}E_{G S} \\
(\mathrm{MeV})\end{array}$ & $\begin{array}{c}E_{G S} / A \\
(\mathrm{MeV})\end{array}$ & $\begin{array}{c}\langle\underset{\sim}{\sim}\rangle \\
(\mathrm{MeV})\end{array}$ & $\begin{array}{c}\langle\underset{\sim}{\sim}\rangle / A \\
(\mathrm{MeV})\end{array}$ & $\begin{array}{c}R_{r m s} \\
(\mathrm{fm})\end{array}$ & $\begin{array}{c}R_{r m s} \\
(\mathrm{fm})\end{array}$ \\
\hline \hline${ }^{4} \mathrm{He}$ & -28.296 & -7.07 & -28.32 & -7.08 & 1.63 & 1.63 \\
\hline${ }^{12} \mathrm{C}$ & -92.163 & -7.68 & -91.36 & -7.61 & 2.42 & 2.79 \\
\hline${ }^{16} \mathrm{O}$ & -127.62 & -7.98 & -127.7 & -7.98 & 2.73 & 2.84 \\
\hline${ }^{19} \mathrm{~F}$ & -147.80 & -7.78 & -143.3 & -7.54 & & 2.87 \\
\hline${ }^{24} \mathrm{Mg}$ & -198.256 & -8.26 & -196.7 & -8.19 & 2.95 & 3.17 \\
\hline${ }^{27} \mathrm{Al}$ & -224.952 & -8.33 & -216.9 & -8.03 & 2.95 & 3.13 \\
\hline${ }^{28} \mathrm{Si}$ & -236.537 & -8.44 & -238.3 & -8.51 & 3.04 & 3.24 \\
\hline${ }^{32} \mathrm{~S}$ & -271.783 & -8.49 & -273.4 & -8.54 & 3.20 & 3.35 \\
\hline${ }^{40} \mathrm{Ca}$ & -342.056 & -8.55 & -349.0 & -8.72 & 3.50 & 3.44 \\
\hline
\end{tabular}

Table 1: Ground state energies and charge radii in FMD. The experimental binding energies are taken from ref. [36, 37] and the charge radii from ref. [38, 39]. All experimental errors are at most in the last digit.

In fig. 2 and fig. 3 we display the density of different ground states in coordinate and momentum space, respectively. The densities are defined as

$$
\rho_{x}(\vec{x})=\left\langle\vec{x}\left|\stackrel{\rho}{\sim}^{(1)}\right| \vec{x}\right\rangle \quad \text { and } \quad \rho_{k}(\vec{k})=\left\langle\vec{k}\left|\stackrel{\rho}{\sim}^{(1)}\right| \vec{k}\right\rangle
$$

where $\underset{\sim}{\rho}{ }^{(1)}$ is the one-body density operator. The crosses indicate the centres $\vec{r}_{l}$ and $\vec{p}_{l}$ of the wave packets. One should however keep in mind that a Slater determinant is invariant under transformations among the occupied single-particle states. In the coordinate representation the density is integrated over the $z$-direction, the momentum representation is shown as a cut at $k_{z}=0$.

In fig. 2 one sees that the ground states are intrinsically deformed. The ${ }^{12} \mathrm{C}$ nucleus for instance arranges as three $\alpha$-clusters, whereas this $\alpha$-symmetry is broken 


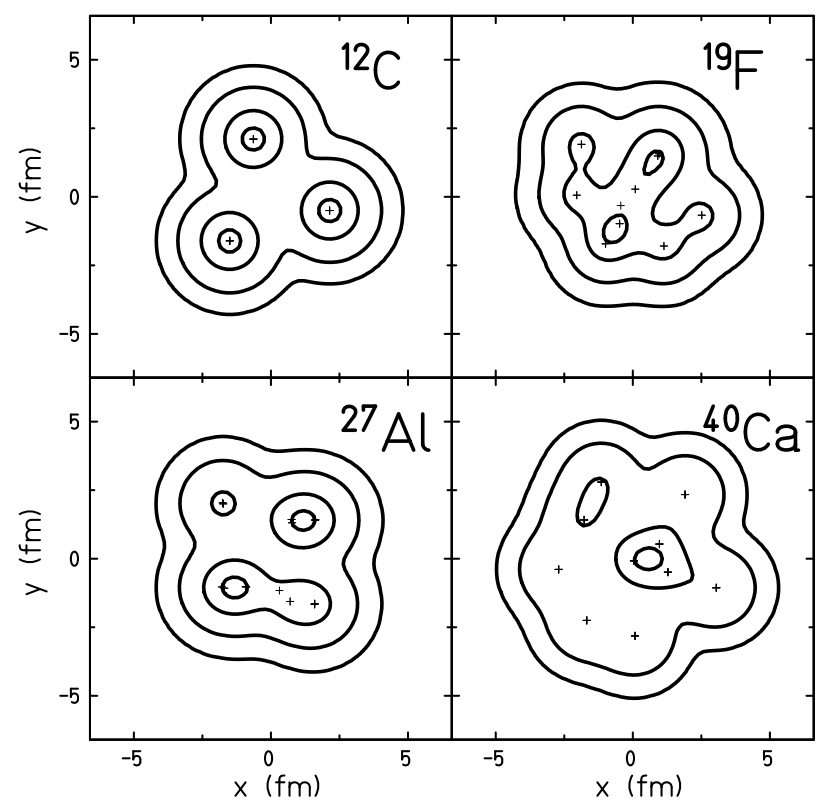

Figure 2: Contour plot of spatial densities integrated over $z$-direction for different ground states. Crosses indicate centroids of wave packets. Contour lines are at $0.9,0.5,0.1$ and 0.01 of the maximum density.

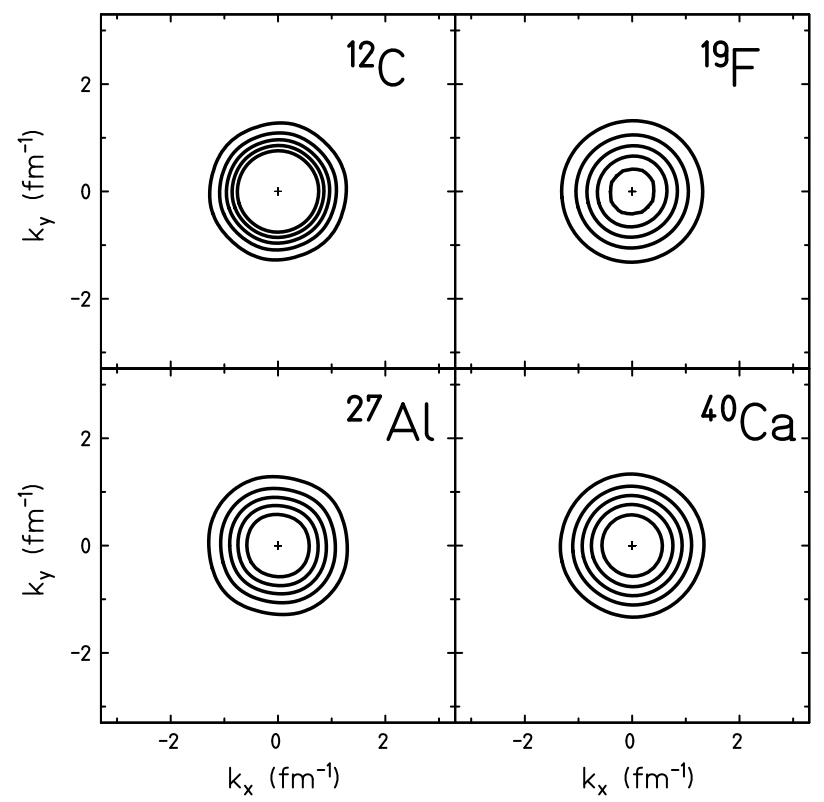

Figure 3: Contour plot of momentum distributions cut at $k_{z}=0$ for different ground states. Crosses indicate centroids of wave packets. Contour lines are at $0.03,0.06,0.09,0.12$ and $0.15 \mathrm{fm}^{3}$. 
in ${ }^{19} \mathrm{~F}$ and ${ }^{27} \mathrm{Al}$. The true ground state, which is an eigenstate of the total spin, is a superposition of all orientations of the intrinsically deformed ground state [28].

The momentum distributions shown in fig. 3 reflect the Fermi motion of the ground states. Although all single-particle wave-packets are stationary (and even centred at $\vec{p}=0$ ) the system possesses Fermi motion. In FMD the Fermi motion is a quantum mechanical zero-point motion and not a random motion of the packet centroids. In the ground state $\vec{r}_{k}$ and $\vec{p}_{k}$ and all other parameters are timeindependent otherwise it would be not the ground state of the system. Using a product state for the many-body system [17, 18, 20, 40, 41, 42, 43] it might be possible to use a momentum dependent Pauli potential in order to mock up the momentum distribution. Nevertheless such a system has the thermodynamic properties of distinguishable particles, which are different from those of a Fermi system especially at low excitations.

\subsection{Shell structure in FMD}

It is not immediately obvious that FMD includes shell-model features like the nodal structure of single-particle orbits since the states are localized in coordinate and momentum space. But due to the invariance of a Slater determinant under linearly independent transformations among the occupied single-particle states, after antisymmetrization, any set of single-particle states which is complete in the occupied phase space is as good as any other. This applies also to non-orthogonal states. To illustrate this we take four one-dimensional real Gaussians with the same real width parameter $a$ and zero mean momentum and displace them by $d=0.75 \sqrt{a}$ (see 1.h.s. of fig. đ). The one-body density can be written in terms of orthonormal states $\left|\psi_{m}\right\rangle$ as

$$
\stackrel{\rho}{\sim}^{(1)}=\sum_{k, l=1}^{A}\left|q_{k}\right\rangle \mathcal{O}_{k l}\left\langle q_{l}\left|=\sum_{m=1}^{A}\right| \psi_{m}\right\rangle\left\langle\psi_{m}\right|
$$

where the orthonormal eigenstates of $\stackrel{\rho}{\sim}^{(1)}$ are given by

$$
\left|\psi_{m}\right\rangle=\sum_{k=1}^{A}\left|q_{k}\right\rangle\left(\mathcal{O}^{\frac{1}{2}}\right)_{k m}
$$

and $\mathcal{O}_{k l}$ is the inverse of the overlap matrix $\left\langle q_{k} \mid q_{l}\right\rangle$. They are displayed on the right hand side of fig. 4 and compared to harmonic oscillator eigenstates (dashed lines). 


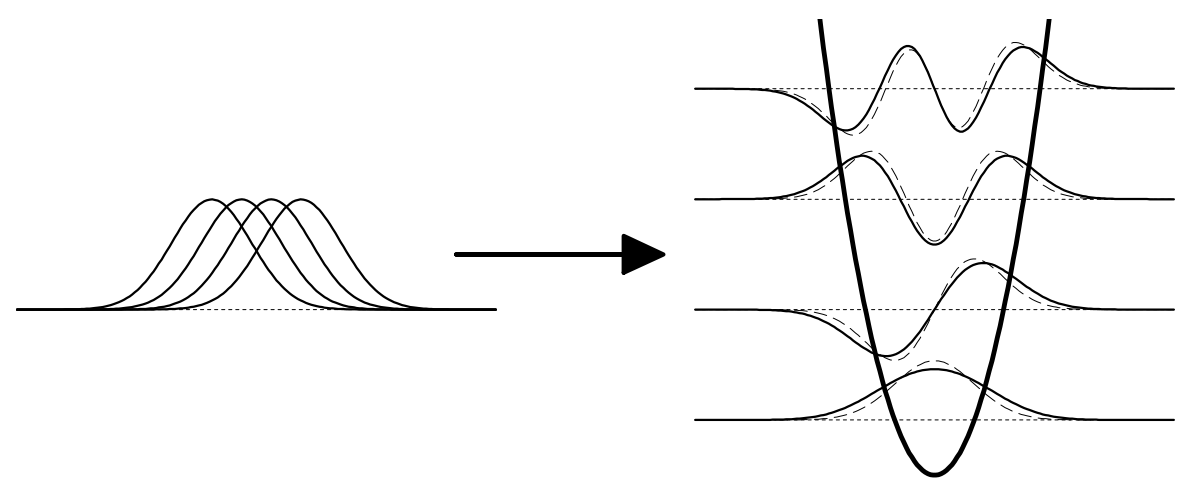

Figure 4: Antisymmetrization of the four Gaussians on the left hand side leads to harmonic oscillator states. Dashed lines are the exact eigenstates of the oscillator.

One sees that the occupied single-particle states $\left|\psi_{m}\right\rangle$ consist of an s-, p- ,d- and an f-state, all very close to harmonic oscillator states. The difference between both sets can be made arbitrarily small by letting $d / \sqrt{a}$ approach zero.

A second example is illustrated in fig. 5, where we consider 100 equally spaced Gaussians in one dimension [44. Again all mean momenta are zero and the width $a$ is real. In the upper part of fig. 5 the width $\sqrt{a}$ is 0.2 of the mean distance $d$ so that the wave packets are well separated. Therefore the spatial density $\rho_{x}$ and the momentum density $\rho_{k}$ are not changed by antisymmetrization. In the lower part the width has been increased to $\sqrt{a}=d$. Without antisymmetrization (dash dotted line) the spatial density is uniform and the momentum distribution is that of a single packet. After antisymmetrization (full lines) one obtains the typical shell model oscillations in coordinate space and a Fermi distribution in momentum space. It is amazing to see how in eq. (48) the superposition of Gaussians by means of the inverse overlap matrices can create a fully occupied momentum state, see for example in fig. 5 the lower right momentum distribution at $k=0.8 k_{F}$, where the individual Gaussians give practically zero probability to measure this momentum. We also calculated the eigenstates of the kinetic energy in the occupied space and got perfect sinusoidal waves.

These two examples illustrate nicely that even localized single-particle states with zero mean momentum build up FMD many-body trial states which describe the harmonic oscillator shell model or even the Fermi motion of a gas of fermions in which plane waves are occupied up to the Fermi momentum. 

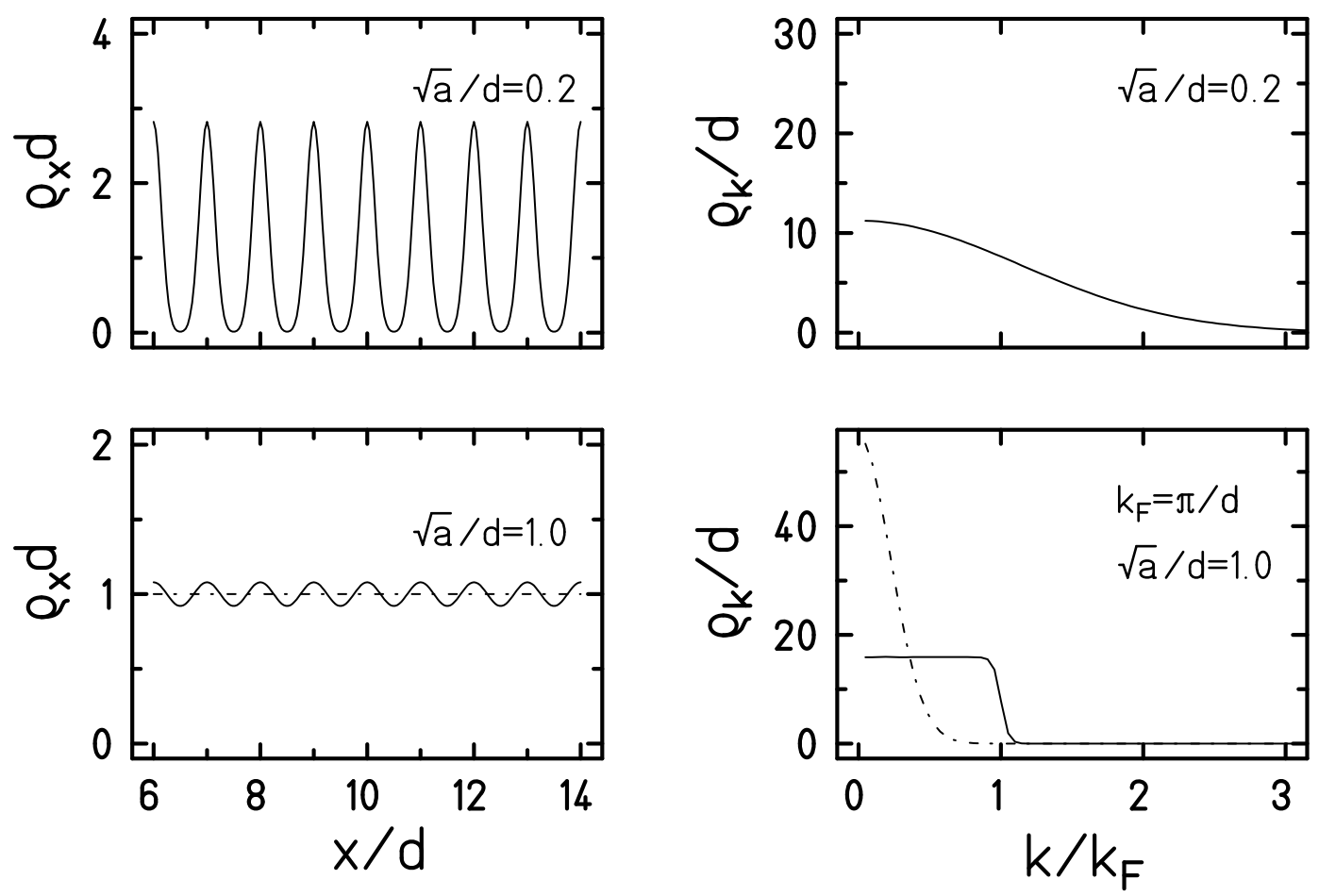

Figure 5: Upper part: section of spatial density of hundred Gaussians (not overlapping in coordinate space) and corresponding momentum distribution (same for all). Antisymmetrization does not change the distribution. Lower part: same as above but for overlapping Gaussians. Full line with antisymmetrization, dashdotted line without. For details see text.

If one wants to simulate this effect by a "Pauli potential", disregarding the momentum distribution in each wave packet, the resulting ground state momentum distribution is unsatisfactory [24].

\section{Heavy ion reactions}

It is the aim of Fermionic Molecular Dynamics to describe the phenomena seen in heavy-ion collisions at laboratory energies of $E \lesssim 200 \mathrm{AMeV}$, as there are fusion, incomplete fusion, dissipative reactions, evaporation of nucleons and fragmentation. In order to gain a predictive power that goes beyond global phenomena dominated by conservation laws three basic conditions are indispensable. 
The first condition concerns the ground state properties. Many observables like for instance fragment multiplicities depend strongly on ground state energies and radii. Thermodynamic properties like the specific heat are related to the exchange symmetry of the many-body state. Equilibration is strongly influenced by the mean free path of the constituents, which is usually much larger for Fermi systems than for classical systems.

The second condition is that the trial state has to have the necessary degrees of freedom for the phenomena one wants to account for. Mean positions and momenta of the single-particle wave-packets are obvious degrees of freedom. For reasons of simplification the width is often chosen as real and time independent, but it could be shown that this degree of freedom is not only useful to reproduce the free motion exactly, but is needed to describe evaporation of nucleons and fragmentation of nuclei [28, 45].

The third important condition, which is related to the second, refers to symmetries. The deterministic equations of motion of FMD preserve all symmetries in the initial state under which the Hamilton operator is invariant. It is therefore desirable to use a Hamilton operator that breaks as many symmetries as possible. This Hamilton operator should not only contain a central potential, as it is the case in the present calculations, but also spin-orbit and tensor interactions, which are expected to break spin symmetries of the ground states during the dynamics. In addition short range correlations have to be considered, which lead to more momentum transfer in the reaction. These short range correlations are responsible for hard collisions of nucleons, a role that is played by the fluctuating collision term in QMD or AMD. First steps into this direction are already taken 46.

The model is chosen to be a molecular dynamics model because it addresses the large fluctuations observed especially in multifragmentation reactions. In the model the molecular aspect is expressed through the localization of single-particle wave-packets; this can be regarded as a quantization of the particle number at any time. It means, fragments have always integer particle number, in contrast to timedependent Hartree-Fock, see for instance [47, 48]. Another aspect is that the FMD single-particle wave-packets are not allowed to split, therefore tunneling through a barrier cannot be described. But this quantal process is of less importance in fragmentation reactions because it takes much longer than the involved time scales. 
The equations of motion of FMD are deterministic, therefore the event ensemble does not arise from random fluctuations of the collision term during the time evolution, but from the average over all orientations of the intrinsically deformed ground states. Although these orientations superimpose coherently in the true ground state their relative phases are randomized during the collision so that they add up incoherently in the exit channels. The same holds true for the summation over different impact parameters [28, 49].

\subsection{Deeply inelastic reactions}

As a first example for heavy-ion collisions described with FMD a dissipative reaction is presented. The reaction of ${ }^{19} \mathrm{~F}$ and ${ }^{27} \mathrm{Al}$ at a laboratory energy of $5.9 \mathrm{AMeV}$ was investigated at the SMP Tandem accelerator in Catania [50]. At this low energy the reaction is dominated by two processes. For impact parameters $b$ up to about $5 \mathrm{fm}$ the system fuses completely, for larger $b$ the reaction shows the typical dissipative phenomena of deeply inelastic collisions. The experiment focussed on the latter process. In order to understand the reaction a view on the nucleus-nucleus potential

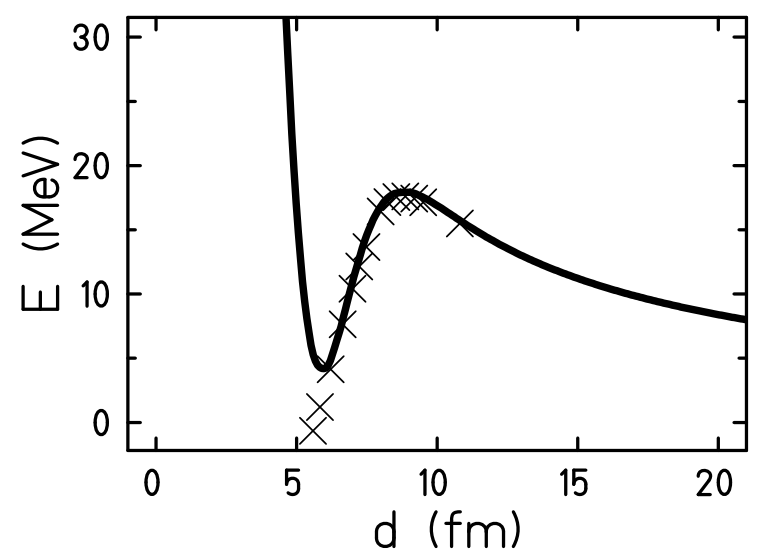

Figure 6: ${ }^{19} \mathrm{~F}-{ }^{27} \mathrm{Al}$ static nucleus-nucleus potential. The solid line shows the FMD result using the nucleon-nucleon interaction (37) and frozen ground states, the crosses indicate the parameterization by Krappe, Nix and Sierk.

might be helpful. In fig. 6 the solid line displays the total energy of the ${ }^{19} \mathrm{~F}-27 \mathrm{Al}$ system (less the ground state energies) as a function of the distance between the 
two centres of mass as it arises from the two-body interaction (37) and (40). The many-body state of the two nuclei was taken to be the respective ground states and not changed as a function of the distance. Therefore, antisymmetrization induces a strong repulsion below $d=5 \mathrm{fm}$. Above $d=5 \mathrm{fm}$ this static interaction compares nicely to the parameterization of the nucleus-nucleus interaction by Krappe, Nix and Sierk [51], which was adjusted to bulk properties of nuclei and successfully applied in earlier studies of heavy-ion dynamics, e.g. in ref. [5].
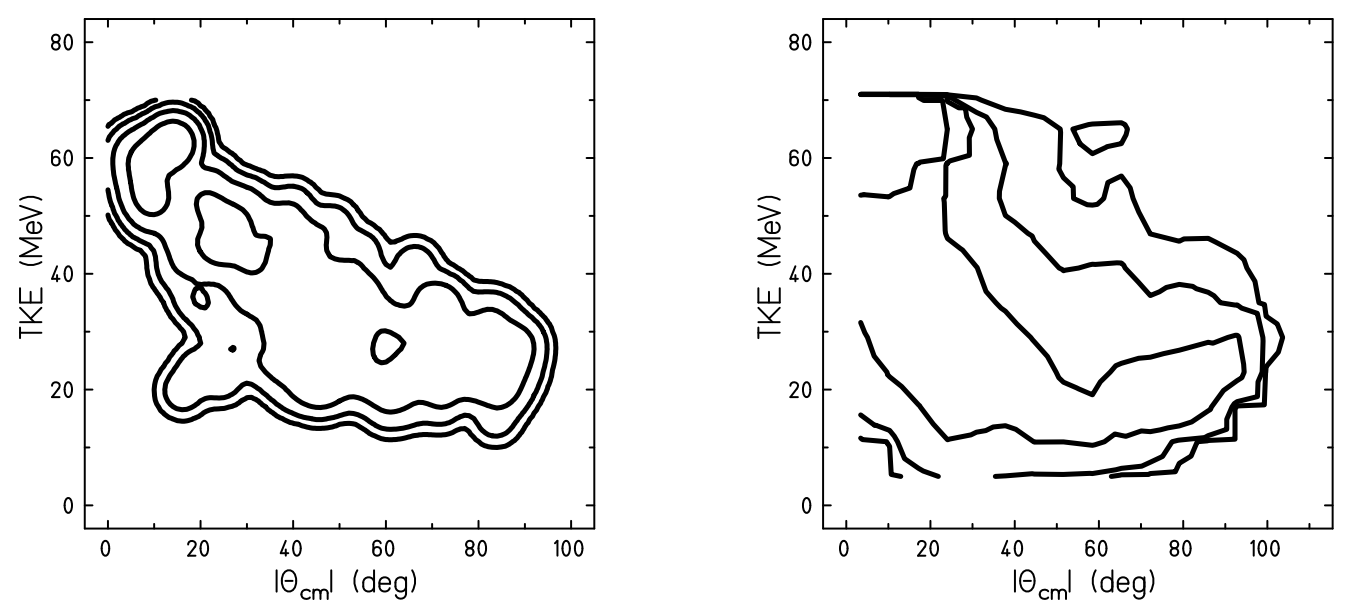

Figure 7: $\quad d^{2} \sigma /\left(d \theta_{\mathrm{cm}} d \mathrm{TKE}\right)$ for ${ }^{19} \mathrm{~F}-27 \mathrm{Al}$ collisions at $5.9 \mathrm{AMeV}$ : FMD calculations are displayed on the l.h.s., experimental results on the r.h.s.. Subsequent contours differ by a factor of 5 .

In the impact parameter range of $4.8 \mathrm{fm} \leq b \leq 10 \mathrm{fm} 509$ events were generated with FMD. The results were filtered with the experimental angular acceptance $3^{\circ} \leq$ $\theta_{\mathrm{Lab}} \leq 54^{\circ}$. Below $b=4.8 \mathrm{fm}$ the system was fusing.

Figure 7 shows the double differential cross section $d^{2} \sigma /\left(d \theta_{\mathrm{cm}} d \mathrm{TKE}\right)$ as a function of the centre of mass scattering angle $\theta_{\mathrm{cm}}$ and the total kinetic energy TKE of the two scattered nuclei. On the left hand side the FMD calculations are presented as a contour plot. Each event $\left(\theta_{\mathrm{cm}}\right.$, TKE) contributes as a Gaussian $\left(\Delta\left|\theta_{\mathrm{cm}}\right|=2^{\circ}\right.$ and $\Delta \mathrm{TKE}=2 \mathrm{MeV}$ ) in order to smoothen the distribution. For impact parameters smaller than the one leading to a grazing collision $\left(\left|\theta_{\mathrm{cm}}\right| \approx 15^{\circ}\right.$, TKE $\left.\approx 65 \mathrm{MeV}\right)$ the approaching nuclei feel the nuclear attraction, $\theta_{\mathrm{cm}}$ gets smaller and becomes negative. The total kinetic energy TKE is decreasing due to internal excitation of the nuclei. The major part of the cross section is observed at negative angles. For 
even smaller impact parameters the nuclei stick together so long that they appear at positive angles again. One observes a flat distribution in $\theta_{\mathrm{cm}}$ at the Viola energy of $\mathrm{TKE} \approx 20 \mathrm{MeV}$. On the right hand side the experimental result is given. Comparing both sides of fig. 7 one sees that FMD is capable to describe the dissipative character of the reaction dynamics.
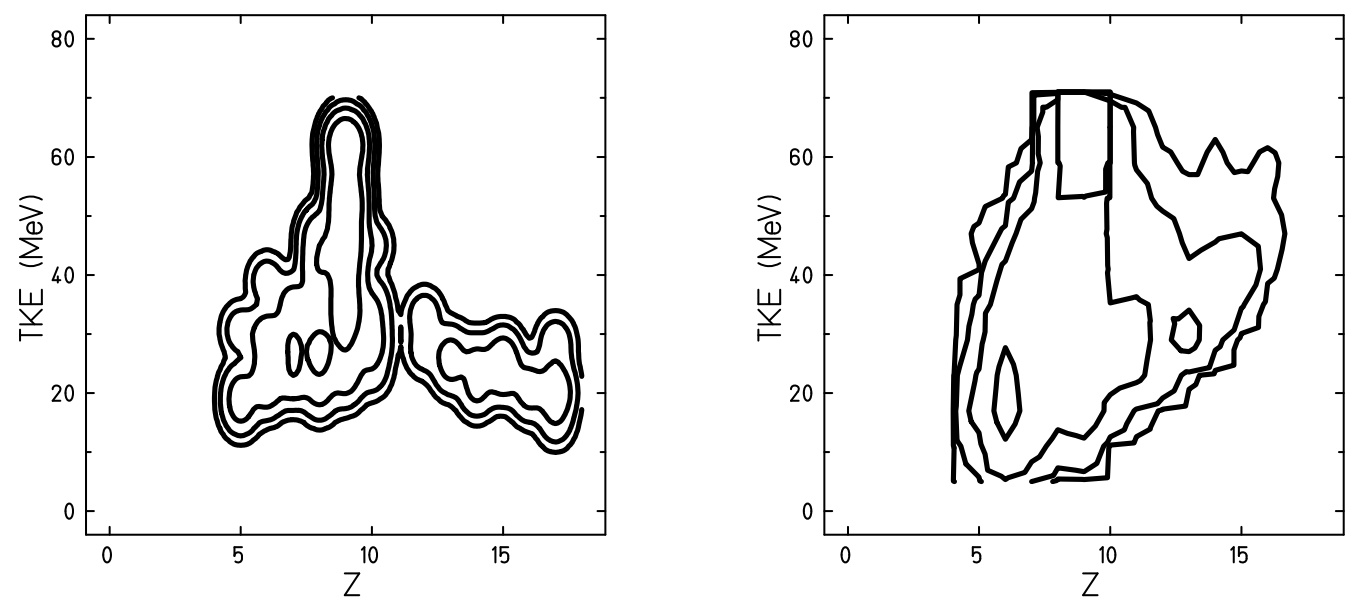

Figure 8: Diffusion plot $d^{2} \sigma /\left(d Z d\right.$ TKE) for ${ }^{19} \mathrm{~F}-{ }^{27} \mathrm{Al}$ collisions at $5.9 \mathrm{AMeV}$ : FMD calculations are displayed on the l.h.s., experimental results on the r.h.s.. Subsequent contours differ by a factor of 5 .

The diffusion plot $d^{2} \sigma /(d Z d$ TKE), fig. 8, samples fragments in the mentioned angular range of $3^{\circ} \leq \theta_{\mathrm{Lab}} \leq 54^{\circ}$, which are mostly projectile like. For the experiment, r.h.s. of fig. 8, the data are limited by an energy threshold seen at low TKE and high $Z$, which is not imposed on the analysis of the FMD simulations. The FMD events $(Z, \mathrm{TKE})$ are smoothened with $\Delta Z=0.5$ and $\Delta \mathrm{TKE}=2 \mathrm{MeV}$.

Both contour plots, FMD (1.h.s.) and experiment (r.h.s.), show the typical broadening of the charge distribution with increasing dissipated energy and the lack of charge drift for TKE above the Viola energy of TKE $\approx 20 \mathrm{MeV}$ for the completely relaxed events. A drift to smaller $Z$ values, which means that the ${ }^{19} \mathrm{~F}$ nucleus is giving away nucleons to the ${ }^{27} \mathrm{Al}$, is seen in the measured and the calculated cross sections for large energy losses where all the initial kinetic energy is dissipated and the reaction lasts very long.

Figure 9 presents the second part of the diffusion plot for $Z \geq 12$ which has 


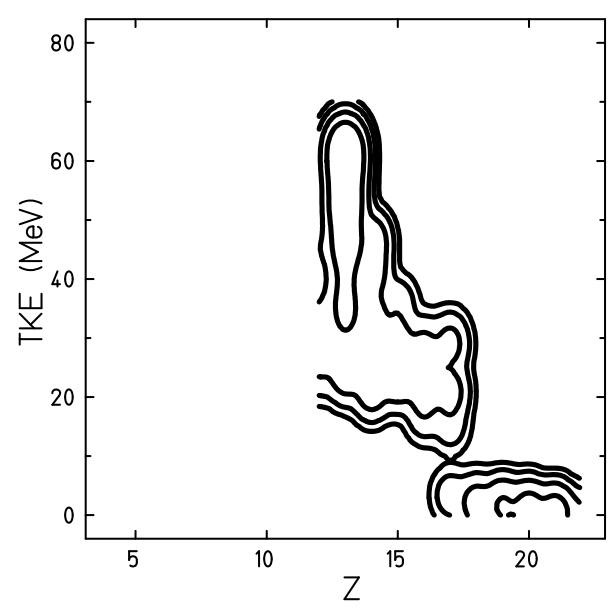

Figure 9: Diffusion plot $d^{2} \sigma /(d Z d \mathrm{TKE})$ for ${ }^{19} \mathrm{~F}-27 \mathrm{Al}$ collisions at $5.9 \mathrm{AMeV}$ calculated with FMD for target like fragments $(Z \geq 12)$. Subsequent contours differ by a factor of 5 .

not been measured. Besides the deeply inelastic reactions one now also sees at TKE values close to zero the evaporation residues from fusion reactions with charges ranging from $Z=16$ to the total charge of $Z=22$ with a maximum around $Z=20$. Here one should note that the FMD time evolution was followed only up to $1200 \mathrm{fm} / c$ so that the loss of charges due to evaporation was not complete yet. Nevertheless the broad distribution of evaporation residues shows that FMD is not unrealistic in this respect.

\subsection{Multifragmentation}

In this section the same system of ${ }^{19} \mathrm{~F}$ and ${ }^{27} \mathrm{Al}$ is investigated, but at an energy of $32 \mathrm{AMeV}$ where multifragmentation is expected.

How multifragmentation happens in heavy ion collisions is still a matter of debate. Explanations reach from nucleation over self organization, spinodal decomposition to cold break-up and survival of initial correlations. For an overview see ref. [52]. A key question is the time scale of the reaction. Slow processes like nucleation or self organization are hindered if the expansion of the whole system is too fast. Another issue is the relaxation time for thermal and chemical equilibrium 
which is important when statistical models are used to explain multifragmentation [53, 54, 55].

If one considers the decay of excited spectator matter which has not been compressed one expects a slow expansion so that there might be enough time for global equilibration. On the other hand the excitation energies are not so high, so that the mean free path, due to the Pauli principle, is still not small compared to the diameter of the nuclear system. Therefore it is not obvious that global thermal equilibrium is achieved.

For the participant matter the compression is much stronger and the excitation energy much higher. This provides a short mean free path, but the whole system is expanding and cooling fast so that the time available for equilibration is rather short and it is questionable if there are enough collisions to ensure local equilibrium until freeze out.

It is very difficult to measure temperature and flow profiles [56, 57, 58] and even harder or impossible to infer experimentally on the time scale of the evolution of the system. Therefore, microscopic transport models which do not assume equilibration are needed for a better understanding. These models should go beyond the mean field approach, which is a kind of equilibrium assumption in itself, so that in principle they are capable to describe many-body correlations like the formation of fragments. QMD, AMD and FMD are molecular dynamics models which assert this claim. How equilibrium is achieved can then be studied by comparing distributions, for example of mass, charge, kinetic energy etc, with equilibrium distributions.

FMD calculations show that correlations play an important role. Since the time evolution of FMD is deterministic, correlations or symmetries can be broken only either in the ground state, e.g. in ${ }^{27} \mathrm{Al}$ there is no $\alpha$-symmetry, but in ${ }^{28} \mathrm{Si}$ there is, or during the time evolution if the Hamilton operator breaks these symmetries dynamically. In models like AMD or QMD a randomly fluctuating collision term, which models the short-range repulsion as a Langevin force, destroys existing correlations and symmetries. We do not want to follow this line, because it is unknown to which extent correlations of the initial state survive during the collision and it even might be an important mechanism for cluster formation in nature.

In the previous section we investigated beam energies of about $6 \mathrm{AMeV}$ which 


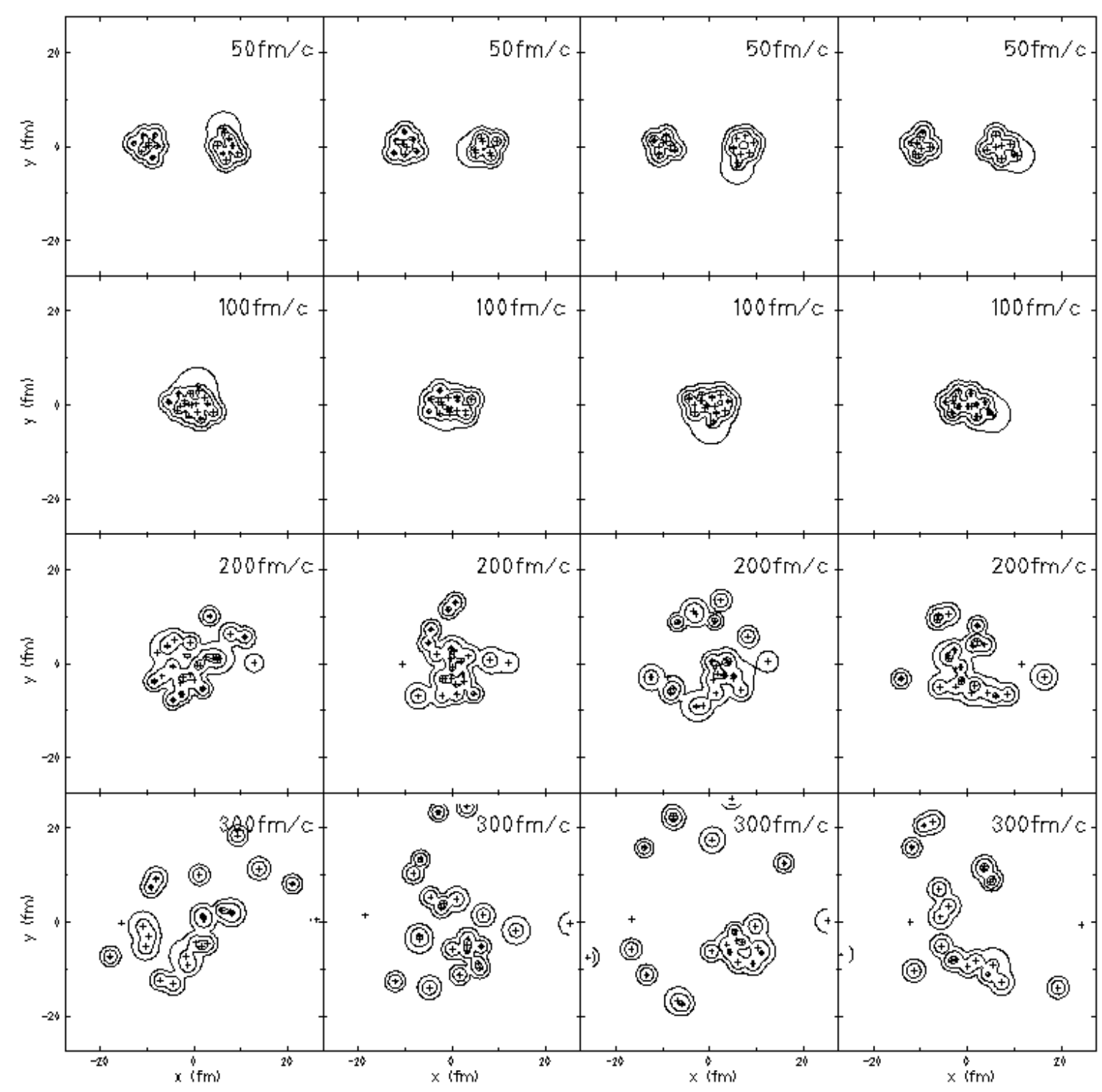

Figure 10: ${ }^{19} \mathrm{~F}-{ }^{27} \mathrm{Al}$ collisions at $32 \mathrm{AMeV}, b=0.5 \mathrm{fm}$ : the one-body density in coordinate space integrated over $z$ is shown. The contour lines depict the density at $0.01,0.1,0.5 \mathrm{fm}^{-2}$.

led to dissipative reactions with two out-coming nuclei with about the same mass number as in the entrance channel. The energy was not high enough to break neither the fused nor the scattered nuclei into pieces.

Now we chose an energy of $32 \mathrm{AMeV}$ that corresponds to a relative velocity between the nuclei of about the Fermi velocity. One expects that at this large collective velocity a common mean field cannot be established any more. 


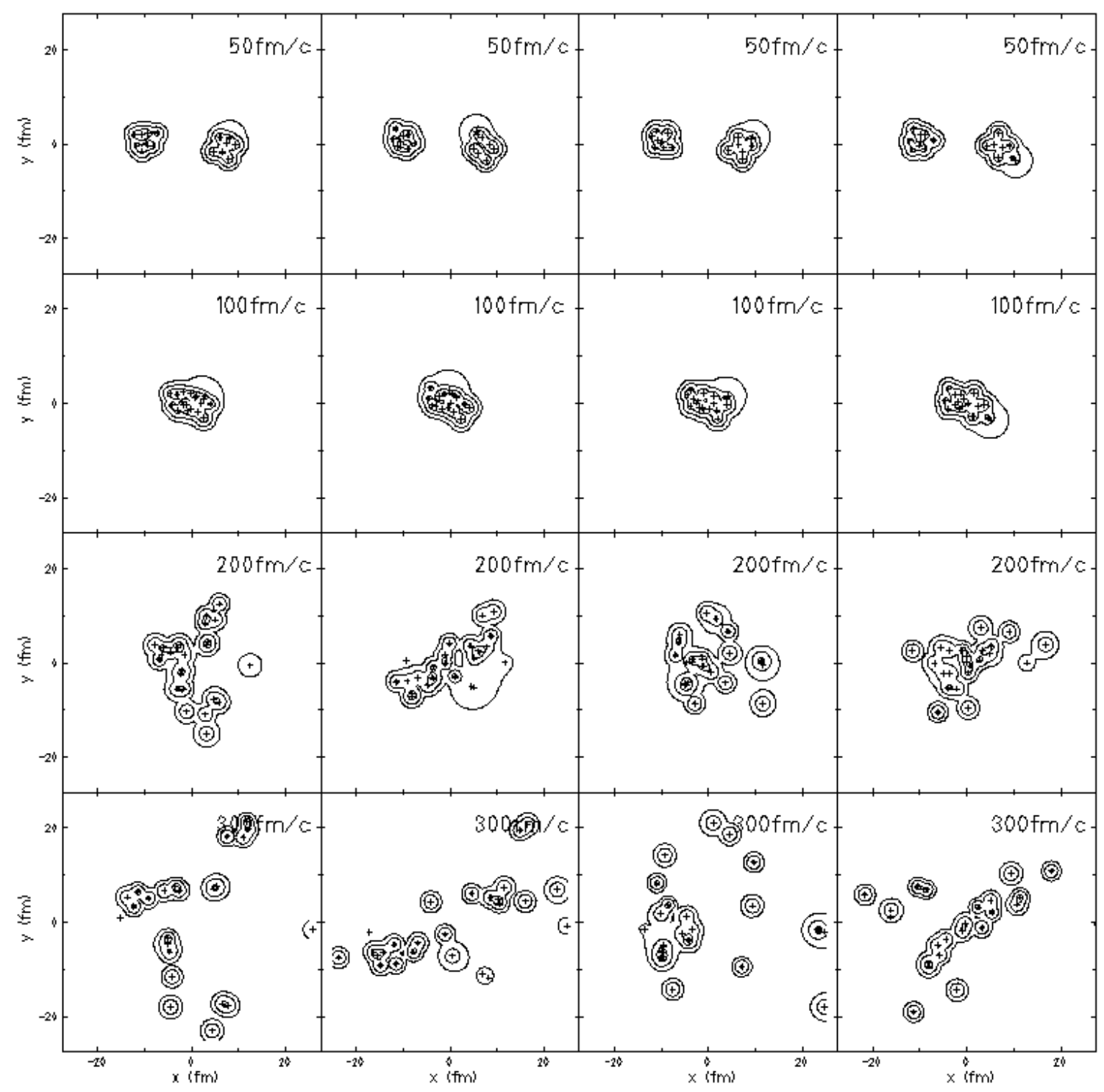

Figure 11: ${ }^{19} \mathrm{~F}-27 \mathrm{Al}$ collisions at $32 \mathrm{AMeV}, b=1.5 \mathrm{fm}$ : for explanation see fig. 10 .

The following figures show a variety of events as contour plots of the one-body density in coordinate space. This density is integrated over the $z$-direction. Figure 10, 11 and 12 present runs at different impact parameters. Within a figure different columns show runs with initial states that differ in the orientation of the intrinsically deformed ground states. Figure 10 presents four time evolutions at an impact parameter of $b=0.5 \mathrm{fm}$. The time is given in the upper right corners. One sees that at this impact parameter and energy a rather long living source is created 


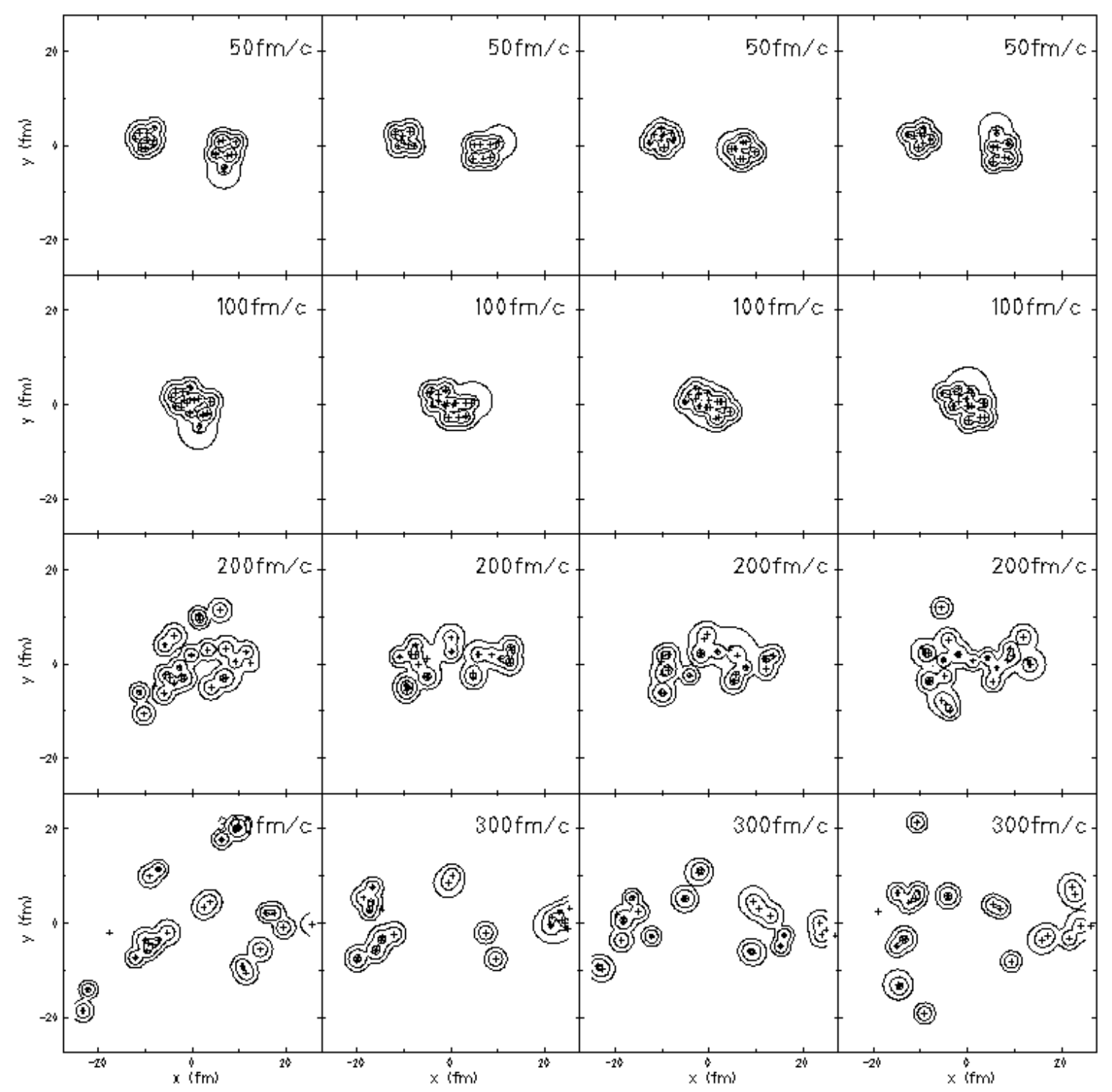

Figure 12: ${ }^{19} \mathrm{~F}-27 \mathrm{Al}$ collisions at $32 \mathrm{AMeV}, b=2.5 \mathrm{fm}$ : for explanation see fig. 10.

which fragments into pieces of all sizes. In fig. 11 for a higher impact parameter of $b=1.5 \mathrm{fm}$ the situation is similar but the source is stretched. Figure 12 finally shows runs with an impact parameter of $b=2.5 \mathrm{fm}$ where the angular distribution of the fragments is more forward-backward peaked than for the central events where it is isotropic.

Analyzing the time evolution of a cluster one sees that the correlations between the wave packets which finally compose the fragment can be followed back for rather 
long time.

In the ground state the wave packets arrange in phase space such that the total energy is minimized. This FMD ground state is an intrinsic state in which the relative positions and orientations of the wave packets reflect many-body correlations. If one destroys these correlations by randomly moving all position parameters $\vec{r}_{k}$ by only $0.2 \mathrm{fm}$, which leaves the one-body density almost unchanged, a ${ }^{56} \mathrm{Fe}$ nucleus for example achieves already $90 \mathrm{MeV}$ excitation energy. A typical $Q$-value to break up lighter fragments (up to about ${ }^{16} \mathrm{O}$ ) into smaller clusters is $10 \mathrm{MeV}$. Therefore, for not too high excitation energies where the mean free path between hard nucleonnucleon collisions is not yet small compared to the diameter of the fragment, one expects the many-body correlations which characterize a bound cluster to survive to a large extend. Since the nucleons are indistinguishable one can of course not say which nucleon from the projectile or target ends up in a given final fragment. Even the wave packets sometimes exchange rapidly $\vec{r}$ and $\vec{p}$, reminding of a Landau-Zener crossing.

It is also interesting to note that in most of the runs several $\alpha$-particles are created (small spheres in the plots), which is in accord with experimental findings at a similar energy of $E=35 \mathrm{AMeV}$ in ${ }^{40} \mathrm{Ca}-{ }^{40} \mathrm{Ca}$ collisions 59.

At this point a few remarks concerning the time-dependent width might be in place, since this degree of freedom is used exclusively in FMD, but not in AMD or QMD and led to a discussion about its role [35, 60].

It was shown not only in our own investigations [45, 44, 28], but also in AMD calculations 61, 62, 63], that a time evolution with fixed width parameters does not produce evaporation or fragmentation. The reason is that a fixed width implies a zero-point energy in each packet of about $\varepsilon_{k i n}=3 /\left(4 m_{N} a_{R}\right) \approx 10 \mathrm{MeV}$. Inside the nucleus this energy is part of the kinetic energy in the Fermi motion. Outside the nucleus the zero-point energy of an emitted particle is determined by the amount of its localization at the end of the emission process. If the width is kept fixed the nucleon carries besides its mean energy always these additional $10 \mathrm{MeV}$, which is much more than the experimentally observed $2 \cdots 3 \mathrm{MeV}$ and thus a wave packet with fixed width has little probability to escape.

Compared to that a wave packet with a dynamical width escapes from an excited 
nucleus by spreading in coordinate space [44, which has two effects: first, the large spatial extend reduces the overlap with the other nucleons and the negative potential energy tends to zero, second, the positive zero-point energy also becomes small so that the sum of both need not change too much during the emission. Classically spoken, a particle leaving the nucleus has to climb up the potential wall at the surface loosing almost all of its kinetic energy before escaping. FMD gives values of about $2 \mathrm{MeV}$ for the kinetic energy of evaporated particles in accord with experimental findings.

For fragmentation the dynamical variation of the width parameters is also needed. Here a change in the width parameters allows the density to notch so that it may break.

In AMD this lack of evaporation and fragmentation is removed by a subtraction of spurious zero-point energy w62 which also takes care of the localization energy in the center of mass motion of the fragments.

\section{$5 \quad$ Statistical properties of FMD}

Fragmentation reactions show large fluctuations, for example in the mass distribution, which are beyond an ensemble averaged mean-field treatment. In this context it is important to understand the statistical properties of molecular dynamic models especially at low temperatures [29].

There are two aspects. One concerns the thermostatic properties of a molecular dynamic model where the attribute thermostatic refers to the properties of the static canonical statistical operator, which are contained in the partition function $Z(T)=\operatorname{Tr}(\exp \{-\underset{\sim}{H} / T\})$. Once the partition function is calculated within a given model, its thermostatic properties can be deduced by standard methods like partial derivatives of $\ln Z(T)$ with respect to temperature $T$ or other parameters contained in the Hamilton operator $\underset{\sim}{\sim}$.

In the case of Fermionic Molecular Dynamics the trace in the partition function can be evaluated exactly because the model is based on antisymmetric many-body states which form an over-complete set covering the whole Hilbert space. Also the states of Antisymmetrized Molecular Dynamics (AMD) [29, 61] provide a represen- 
tation for the unit operator. As the calculation of the trace does not depend on the representation all thermostatic properties like Fermi-Dirac distribution, specific heat, mean energy as a function of temperature etc. ought to be correct and fully quantal using FMD or AMD trial states.

The issue of this section is more the other and even more important aspect, namely the dynamical behaviour of a molecular dynamics model. A dissipative system which is initially far from equilibrium is expected to equilibrate towards the canonical ensemble. The simulation of such a system within the model provides a crucial test of its thermodynamic behaviour.

The time--evolved FMD state is in general not the exact solution of the Schrödinger equation, so the correct thermostatic properties do not a priori guarantee correct thermodynamic properties. In other words the question is: does the FMD state as a function of time explore the Hilbert space according to the canonical weight?

Since the parameters of the single-particle wave packets follow generalized Hamilton equations of motion, one is tempted to infer that the dynamical statistical properties might be classical [64, 65]. This conjecture, that classical equations of motion always imply classical statistics, is disproved by the following examples, in which we compare time-averaged expectation values of wave-packet molecular dynamics with the equivalent ones of the canonical ensemble at the same excitation energy.

Within Fermionic Molecular Dynamics we study the equilibration of four identical fermions enclosed in a one dimensional harmonic oscillator. The particles interact by a weak repulsive two-body potential which is necessary to convert the integrable harmonic oscillations into chaotic motion. The important result is that the initial many-body state, which is far from equilibrium, approaches the canonical ensemble with Fermi-Dirac statistics in an ergodic sense. The time-averaged occupation numbers of the harmonic oscillator eigenstates are practically identical with the Fermi-Dirac distribution of the canonical ensemble, provided the canonical ensemble is taken at the time-independent mean excitation energy of the many-body state.

When distinguishable particles, which are described by a product state of wave packets, are considered, the molecular dynamic equations for the parameters of the wave packets lead to a Boltzmann distribution for the occupation numbers of the 
single-particle eigenstates.

A further important result is, that the use of time averages provides us with a tool for establishing relations between well-defined quantities of a molecular dynamic model such as excitation energy and statistical quantities like temperature. This will be done in subsection 5.3 where we investigate excited nuclei and the nuclear liquid-gas phase transition which is of great experimental [56, 57, 66] and theoretical interest [54, 67, 68, 69, 70, 71, 72, 73.

\subsection{Thermostatic properties}

In order to describe the thermostatic properties of a given system by means of model states exactly, it is necessary that these model states span the whole Hilbert space. The question, whether the thermostatic properties of FMD are correct, therefore reduces to the question whether its model states are complete. Starting from coherent states the following short explanation proofs that this is the case.

\subsubsection{Completeness relation with coherent states}

Coherent states $|\vec{z}\rangle$ which are defined as the eigenstates of the harmonic oscillator destruction operator $\underset{a}{\sim}$

$$
\underset{\sim}{\sim}|\vec{z}\rangle=\vec{z}|\vec{z}\rangle, \quad \underset{\sim}{H_{\mathrm{HO}}}=\omega\left(\underset{\vec{a}}{\sim} \stackrel{\vec{a}}{\sim}+\frac{3}{2}\right)
$$

form an over-complete set of states. Their completeness relation reads

$$
\begin{aligned}
\mathbb{1}^{(1)} & =\int \frac{\mathrm{d}^{3} \operatorname{Re}(z) \mathrm{d}^{3} \operatorname{Im}(z)}{\pi^{3}} \frac{|\vec{z}\rangle\langle\vec{z}|}{\langle\vec{z} \mid \vec{z}\rangle} \\
& =\int \frac{\mathrm{d}^{3} r \mathrm{~d}^{3} p}{(2 \pi)^{3}} \frac{|\vec{r}, \vec{p}\rangle\langle\vec{r}, \vec{p}|}{\langle\vec{r}, \vec{p} \mid \vec{r}, \vec{p}\rangle} \\
& =\int \frac{\mathrm{d}^{3} r \mathrm{~d}^{3} p}{(2 \pi)^{3}} \frac{|q\rangle\langle q|}{\langle q \mid q\rangle},
\end{aligned}
$$

where all three lines are equivalent notations; $|\vec{z}\rangle$ labeling coherent states by their eigenvalue with respect to $\underset{\sim}{\sim}$, the phase space notation $|\vec{r}, \vec{p}\rangle$ labeling the states by their expectations values of the coordinate and momentum operators and $|q\rangle$ being the FMD notation. Coherent states are extensively discussed in ref. [74. 
Since we are dealing with fermions the spin degree of freedom has to be considered and consequently the resolution of unity changes to

$$
\mathbb{1}^{(1)}=\int \frac{\mathrm{d}^{3} r \mathrm{~d}^{3} p}{(2 \pi)^{3}} \sum_{m_{s}} \frac{|q\rangle\langle q|}{\langle q \mid q\rangle}
$$

where the sum runs over the different magnetic quantum numbers $m_{s}$ which are included in the set of parameters denoted by $q$ (see eq. (7)) .

Proceeding one step further the unity operator in the antisymmetric part of the two-particle Hilbert space is the antisymmetric product of two single-particle unity operators

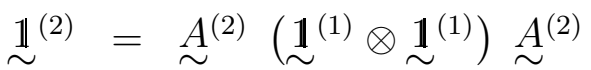

$$
\begin{aligned}
& =\frac{1}{2}\left(1-{\underset{\sim}{P}}_{12}\right)\left(\mathbb{1}^{(1)} \otimes \mathbb{1}^{(1)}\right) \frac{1}{2}\left(1-{\underset{\sim}{P}}_{12}\right) \text {, }
\end{aligned}
$$

which may be expressed with antisymmetric two-body states $\left|q_{1}, q_{2}\right\rangle_{a}$ as

$$
\begin{aligned}
& \stackrel{\mathbb{1}}{\sim}^{(2)}=\int \frac{\mathrm{d}^{3} r_{1} \mathrm{~d}^{3} p_{1}}{(2 \pi)^{3}} \sum_{m_{s}(1)} \int \frac{\mathrm{d}^{3} r_{2} \mathrm{~d}^{3} p_{2}}{(2 \pi)^{3}} \sum_{m_{s}(2)} \frac{\left|q_{1}, q_{2}\right\rangle_{a}{ }_{a}\left\langle q_{1}, q_{2}\right|}{\left\langle q_{1} \mid q_{1}\right\rangle\left\langle q_{2} \mid q_{2}\right\rangle} \\
& \text { where } \quad\left|q_{1}, q_{2}\right\rangle_{a}:=\frac{1}{2}\left(\left|q_{1}\right\rangle \otimes\left|q_{2}\right\rangle-\left|q_{2}\right\rangle \otimes\left|q_{1}\right\rangle\right) .
\end{aligned}
$$

Following this line the resolution of unity in the antisymmetric part of the $A$-body Hilbert space is just the antisymmetric product of single-particle unity operators. Be $|\hat{Q}\rangle$ the unnormalized Slater determinant of single-particle states $|q\rangle$ and $|Q\rangle$ the normalized Slater determinant

$$
\begin{aligned}
|\hat{Q}\rangle & =\frac{1}{A !} \sum_{\pi} \operatorname{sgn}(\pi)\left(\left|q_{\pi(1)}\right\rangle \otimes \cdots \otimes\left|q_{\pi(A)}\right\rangle\right) \\
|Q\rangle & =\frac{1}{\langle\hat{Q} \mid \hat{Q}\rangle^{\frac{1}{2}}}|\hat{Q}\rangle .
\end{aligned}
$$

Then the unity operator can be written as the projection of the $A$-body unit operator onto the antisymmetric subspace of the Hilbert space

$$
{\underset{1}{\sim}}^{(A)}=\int \mathrm{d} \mu(Q)|Q\rangle\langle Q|
$$

with a measure

$$
\mathrm{d} \mu(Q)=\langle\hat{Q} \mid \hat{Q}\rangle \prod_{k=1}^{A} \frac{1}{\left\langle q_{k} \mid q_{k}\right\rangle} \frac{\mathrm{d}^{3} r_{k} \mathrm{~d}^{3} p_{k}}{(2 \pi)^{3}} \sum_{m_{s}(k)}
$$


that accounts for antisymmetrization by means of the ratio between the norm of the Slater determinant $\langle\hat{Q} \mid \hat{Q}\rangle$ and the norm of the corresponding product state $\prod_{k=1}^{A}\left\langle q_{k} \mid q_{k}\right\rangle$ (see also 64]). In a sampling where the values of $\vec{r}_{k}$ and $\vec{p}_{k}$ are chosen according to Monte Carlo methods this measure determines the probability to find the state $|Q\rangle$ belonging to this configuration $Q=\left\{\vec{r}_{1}, \vec{p}_{1} ; \vec{r}_{2}, \vec{p}_{2} ; \ldots\right\}$ in Hilbert space. If for example two fermions with the same spin are close in $\vec{r}$ and $\vec{p}$ then this measure is small because the norm $\langle\hat{Q} \mid \hat{Q}\rangle=\operatorname{det}\left\{\left\langle q_{k} \mid q_{l}\right\rangle\right\}$ will be small.

Coherent states are Gaussian wave-packets with fixed width, but the singleparticle states of FMD

$$
\langle\vec{x} \mid q\rangle \propto \exp \left\{-\frac{(\vec{x}-\vec{r})^{2}}{2 a}+i \vec{p} \cdot \vec{x}\right\} \otimes|\chi, \phi\rangle \otimes|\xi\rangle
$$

contain more degrees of freedom, for instance the width parameter $a$, the spin angles $\chi$ and $\phi$ and the isospin-3 component $\xi$. Since coherent states are already complete the additional degrees of freedom $a_{R}$ and $a_{I}$ do not bother. They can be integrated keeping track of the normalization as it is shown in (59) and the isospin is summed over like the spin

$$
\mathbb{1}^{(1)}=\frac{1}{\Omega_{R} \Omega_{I}} \int \frac{\mathrm{d}^{3} r \mathrm{~d}^{3} p}{(2 \pi)^{3}} \sum_{m_{s}, \xi} \int_{\Omega_{R}} \mathrm{~d} a_{R} \int_{\Omega_{I}} \mathrm{~d} a_{I} \frac{|q\rangle\langle q|}{\langle q \mid q\rangle},
$$

where $\Omega_{R}$ and $\Omega_{I}$ denote the intervals the width $a=a_{R}+i a_{I}$ is integrated over.

$$
\int_{\Omega_{R}} \mathrm{~d} a_{R}=\Omega_{R}, \quad \int_{\Omega_{I}} \mathrm{~d} a_{I}=\Omega_{I}
$$

The measure then changes to

$$
\mathrm{d} \mu(Q)=\langle\hat{Q} \mid \hat{Q}\rangle \prod_{k=1}^{A} \frac{1}{\left\langle q_{k} \mid q_{k}\right\rangle} \frac{\mathrm{d}^{3} r_{k} \mathrm{~d}^{3} p_{k}}{(2 \pi)^{3}} \frac{\mathrm{d} a_{R}}{\Omega_{R}} \frac{\mathrm{d} a_{I}}{\Omega_{I}} \sum_{m_{s}(k), \xi(k)} .
$$

\subsubsection{The partition function}

Once the resolution of unity is given in terms of model states the partition function can be evaluated. Eq. (56) is very useful in calculating traces by means of Monte Carlo sampling [64]. For instance the canonical partition function is given by

$$
\begin{aligned}
Z(T) & =\operatorname{Tr}(\exp \{\underset{\sim}{-H} / T\}) \\
& =\int \mathrm{d} \mu(Q)\langle Q|\exp \{-\underset{\sim}{H} / T\}| Q\rangle .
\end{aligned}
$$




\subsubsection{Example}

In the following the above considerations are illustrated with the example of $A$ identical fermions in a common one-dimensional harmonic oscillator potential 46. Starting from the Hamilton operator

$$
\underset{\sim}{\sim}=\sum_{n=1}^{A} \underset{\mathrm{h}}{\sim} \mathrm{HO}(n), \quad \underset{\mathrm{h}}{\stackrel{h^{2}}{\sim}}(n)=\frac{\vec{k}^{2}(n)}{2 m}+\frac{1}{2} m \omega^{2} \stackrel{\vec{x}}{\sim}^{2}(n)
$$

the mean energy of the $A$-fermion system can be derived from the partition function $Z(T)(61)$ as the derivative with respect to $T$

$$
\begin{aligned}
\langle\langle\underset{\sim}{\sim}\rangle\rangle_{\mid T} & =T^{2} \frac{\partial}{\partial T} \ln (Z(T)) \\
& =\frac{\int \mathrm{d} \mu(Q) \mathcal{W}(T) \sum_{m, n}^{A} \mathcal{O}_{n m}(T)\left[T ^ { 2 } \frac { \partial } { \partial T } \langle q _ { m } | \operatorname { e x p } \left\{-\underset{\sim}{\left.\left.h_{\mathrm{HO}} / T\right\}\left|q_{n}\right\rangle\right]}\right.\right.}{\int \mathrm{d} \mu(Q) \mathcal{W}(T)},
\end{aligned}
$$

where two the abbreviations $\mathcal{W}(T)$ and $\mathcal{O}^{-1}(T)$ are introduced as

$$
\begin{aligned}
\mathcal{W}(T) & =\frac{\operatorname{det}\left(\left\langle q_{k}\left|\exp \left\{-\underset{\sim}{\sim} \mathrm{HO}_{\mathrm{O}} / T\right\}\right| q_{l}\right\rangle\right)}{\operatorname{det}\left(\left\langle q_{k} \mid q_{l}\right\rangle\right)}, \\
\left(\mathcal{O}^{-1}(T)\right)_{k l} & =\left\langle q_{k}\right| \exp \left\{-\underset{\sim}{\left.h_{\mathrm{HO}} / T\right\}\left|q_{l}\right\rangle .}\right.
\end{aligned}
$$

The matrix elements can be evaluated in closed form [46]. Figure 13] shows the result of a Monte-Carlo simulation for a system of four identical fermions in a harmonic oscillator with $\hbar \omega=8 \mathrm{MeV}$ by open circles. The solid line shows the same result, but calculating the partition function with eigenstates of the Hamilton operator, which obviously gives the same relation. For comparison the classical dependence is shown as a dashed-dotted line.

\subsection{Canonical versus ergodic ensemble}

Fermionic Molecular Dynamics is a deterministic microscopic transport theory. Given the Hamilton operator and a state $\left|Q\left(t_{0}\right)\right\rangle$ at a certain time $t_{0}$ the state $|Q(t)\rangle$ is known for all times.

Expectation values are well-defined in FMD so that one can easily calculate quantities like the excitation energy of a nucleus or the probability of finding the 


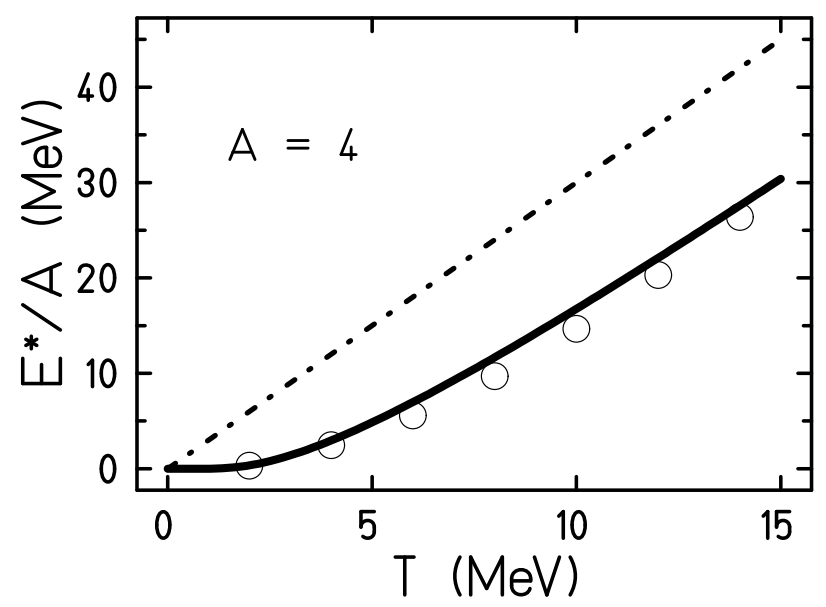

Figure 13: Excitation energy as a function of temperature, calculated with FMD model states (circles) or with eigenfunctions of the harmonic oscillator (solid line). The dashed-dotted line shows the classical result.

system in a given reference state. But it is not obvious how intensive thermodynamical quantities, such as the temperature, might be extracted from deterministic molecular dynamics with wave packets. In classical mechanics with momentumindependent interactions the partition function

$$
\begin{aligned}
& Z_{\text {classical }}(T)=\int \prod_{k=1}^{A} \frac{\mathrm{d}^{3} r_{k} \mathrm{~d}^{3} p_{k}}{(2 \pi)^{3}} \exp \left\{-\frac{1}{T} \mathcal{H}_{\text {classical }}\left(\vec{r}_{1}, \vec{p}_{1}, \cdots\right)\right\} \\
= & \int \prod_{k=1}^{A} \mathrm{~d}^{3} p_{k} \exp \left\{-\frac{1}{T} \sum_{i=1}^{A} \frac{\vec{p}_{i}^{2}}{2 m_{i}}\right\} \times \int \prod_{l=1}^{A} \frac{\mathrm{d}^{3} r_{l}}{(2 \pi)^{3}} \exp \left\{-\frac{1}{T} \mathcal{V}\left(\vec{r}_{1}, \vec{r}_{2}, \cdots\right)\right\}
\end{aligned}
$$

is a product of a term with the kinetic energy and a term containing the interactions. Therefore, the momentum distribution can be used to determine the temperature $T$. In the quantum case eq. (61) has to be employed which does not show this factorization. A simple example for this behaviour is the ground state of the free Fermi gas where finite momenta are occupied, nevertheless the temperature is zero. Another example is the ground state of a nucleus for which the momentum distribution has a smeared out Fermi edge due to the finite size and not because of temperature.

In this section time averaging is compared with a statistical ensemble. If the system is ergodic both are equivalent and statistical properties of molecular dynamics can be evaluated by means of time averaging. 
For this the ergodic ensemble is defined by the statistical operator $\underset{\sim}{R}$ erg as

$$
\underset{R}{\sim} \operatorname{erg}:=\lim _{t_{2} \rightarrow \infty} \frac{1}{\left(t_{2}-t_{1}\right)} \int_{t_{1}}^{t_{2}} \mathrm{~d} t|Q(t)\rangle\langle Q(t)| .
$$

The ergodic mean of an operator $\underset{\sim}{B}$ is given by

$$
\left.\overline{\langle\underset{\sim}{\sim}\rangle_{\mid}}\right|_{\sim \underset{\sim}{\sim}\rangle}:=\operatorname{Tr}(\underset{\sim}{R} \operatorname{erg} \underset{\sim}{\stackrel{B}{\sim}})=\lim _{t_{2} \rightarrow \infty} \frac{1}{\left(t_{2}-t_{1}\right)} \int_{t_{1}}^{t_{2}} \mathrm{~d} t\langle Q(t)|\underset{\sim}{B}| Q(t)\rangle .
$$

In general the statistical operator $\underset{\sim}{R} \operatorname{erg}$ is a functional of the initial state $\left|Q\left(t_{1}\right)\right\rangle$, the Hamilton operator $\underset{\sim}{\mathrm{H}}$ and the equations of motion. If the ergodic assumption is fulfilled, the statistical operator should only depend on $\langle\underset{\sim}{H}\rangle$, which is actually a constant of motion. Thus the average in the ergodic ensemble is always performed at the same expectation value of the Hamilton operator. In our notation this is denoted by the condition " $\langle\underset{\sim}{H}\rangle "$ in eq. (67).

\subsubsection{Canonical ensemble of fermions in a harmonic oscillator}

With the statistical operator of the canonical ensemble for $A$ identical fermions (all spin up) in a one-dimensional common harmonic oscillator potential $\underset{\sim}{H}$ HO given by

$$
\begin{aligned}
& \underset{\sim}{\sim}(T)=\frac{1}{Z(T)} \exp \left\{-\frac{H_{\mathrm{HO}}}{T}\right\} \\
& \underset{\sim}{H_{\mathrm{HO}}}=\sum_{l=1}^{A} \underset{\sim}{\sim}(l), \quad \underset{\sim}{\sim}(l)=\omega \sum_{n=0}^{\infty}\left(n+\frac{1}{2}\right) \underset{\sim}{\stackrel{c}{\sim}} \stackrel{c}{\sim}_{n},
\end{aligned}
$$

the statistical mean of an operator $\underset{\sim}{B}$ is calculated as

$$
\begin{aligned}
& \langle\langle\underset{\sim}{\sim}\rangle\rangle_{T}:=\operatorname{Tr}(\underset{\sim}{R}(T) \underset{\sim}{\sim}) \\
& =\frac{1}{Z(T)} \int \frac{\mathrm{d} r_{1} \mathrm{~d} p_{1}}{2 \pi} \cdots \frac{\mathrm{d} r_{A} \mathrm{~d} p_{A}}{2 \pi}\left\langle\hat{Q}\left|\underset{\sim}{\sim} \exp \left\{-\frac{H_{\mathrm{HO}}}{T}\right\}\right| \hat{Q}\right\rangle \\
& =\frac{1}{Z(T)} \sum_{n_{1}<\cdots<n_{A}}\left\langle n_{1}, \cdots, n_{A}|\underset{\sim}{B}| n_{1}, \cdots, n_{A}\right\rangle \exp \left\{-\frac{E\left(n_{1}, \cdots, n_{A}\right)}{T}\right\} .
\end{aligned}
$$

As already mentioned the FMD states are a representation of the unit operator and hence can be used to calculate traces. For numerical convenience, however, the mathematically identical third line in eq. (69) is used, where $\left|n_{1}, \cdots, n_{A}\right\rangle$ denotes the Slater determinant composed of single-particle oscillator eigenstates $\left|n_{1}\right\rangle, \cdots,\left|n_{A}\right\rangle$ and

$$
E\left(n_{1}, \cdots, n_{A}\right)=\omega \sum_{i=1}^{A}\left(n_{i}+\frac{1}{2}\right)
$$


are the eigenenergies of $\underset{\sim}{H} \mathrm{HO}$. In eq. (69) the subscript $T$ indicates that the average is taken at a constant temperature $T$.

In the following a system of four fermions in a common one-dimensional harmonic oscillator is investigated. The frequency of the oscillator is chosen to be $\omega=0.04 \mathrm{fm}^{-1}$ in order to get a spacing of $8 \mathrm{MeV}$ between the single-particle eigenstates. For the canonical ensemble fig. 14 shows the dependence of the excitation energy on the temperature (l.h.s.) and displays how the lowest eigenstates are occupied in the four-fermion system for five different temperatures (r.h.s.).
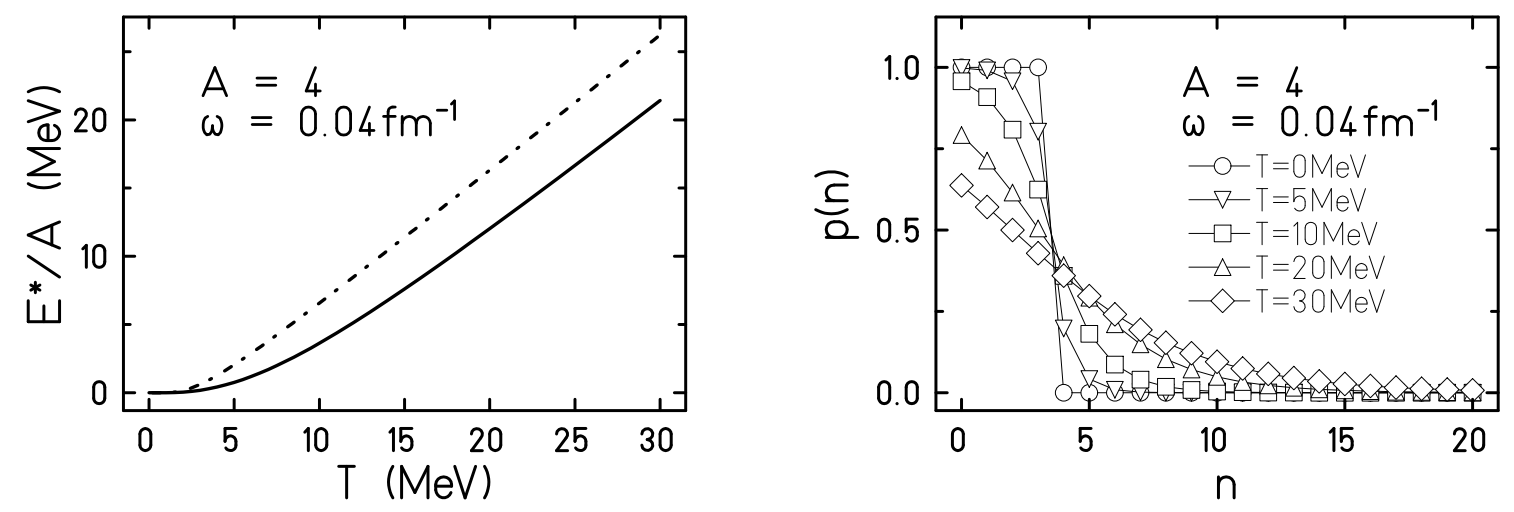

Figure 14: A system of four fermions in a common oscillator described by the canonical ensemble. L.h.s.: Excitation energy as a function of temperature (solid line). The dashed-dotted line shows the result for a product state (Boltzmann statistics). R.h.s.: Occupation numbers $p(n)$ of the oscillator eigenstates for five temperatures (eq. (71)). The lines are drawn as a guide for the eye.

The mean occupation probabilities are given by

$$
p(n)=\left\langle\left\langle\underset{\sim}{\stackrel{c}{\sim} \underset{\sim}{\sim}}{ }_{n}\right\rangle\right\rangle_{T},
$$

where $\underset{\sim}{\stackrel{c}{\sim}}$ denotes the creation operator of a fermion in the oscillator eigenstate $|n\rangle$.

\subsubsection{Ergodic ensemble of fermions in a harmonic oscillator}

In this section the averages of the occupation numbers in the ergodic ensemble are evaluated and compared with those of the canonical ensemble discussed in the 
previous section. As pointed out already, in Fermionic Molecular Dynamics the time evolution of Gaussian wave packets in a common oscillator is exact, and thus the occupation probabilities of the eigenstates of the Hamilton operator do not change in time. In order to equilibrate the system a repulsive short-range interaction $\underset{\sim}{V_{I}}$ is introduced. The strength of the interaction is chosen such that the resulting matrix elements of $\underset{\sim}{V_{\text {I }}}$ are small compared to the level spacing $\omega$ and the excitation energy $E^{*}$. The contribution of $\left\langle\underset{\sim}{V_{I}}\right\rangle$ to the total energy is of the order of $0.1 \ldots 1.0 \mathrm{MeV}$.

The initial state is prepared in the following way. Three wave packets with a width of $a=1 / m \omega$ are put close to the origin at $x=(-d, 0, d)$ - with $d=0.5 / \sqrt{m \omega}$ - whereas the fourth packet with the same width is pulled away from the centre in order to obtain the desired energy. As the mean momenta are all zero, the excitation is initially only in potential energy which has to be converted into thermal energy by means of the small interaction $\underset{\sim}{V}$.

The initial system, which is far from equilibrium, is evolved over about 2000 periods of the harmonic oscillator $(2 \pi / \omega=157 \mathrm{fm} / \mathrm{c})$. The equilibration time is rather large as we are using a very weak interaction in order not to introduce correlations which would destroy the ideal gas picture implied in the canonical ensemble (68) of non-interacting particles. The time averaging of the occupation numbers (72) starts at time $t_{1}=10000 \mathrm{fm} / \mathrm{c}$ in order to allow a first equilibration.

$$
{\overline{\left\langle\underset{\sim}{\left.c_{n}^{+} \underset{\sim}{\sim}{ }_{n}\right\rangle}\right.}}_{\langle\underset{\sim}{\sim}\rangle}=\lim _{t_{2} \rightarrow \infty} \frac{1}{\left(t_{2}-t_{1}\right)} \int_{t_{1}}^{t_{2}} \mathrm{~d} t\langle Q(t)|\underset{\sim}{\underset{\sim}{\sim}} \underset{\sim}{\stackrel{c}{\sim}}| Q(t)\rangle
$$

Figure 15 gives an impression of how the occupation numbers evolve in time. The part to the left shows the time evolution without interaction which is just a unitary transformation in the one-body space. Thus the occupation numbers do not change in time although the wave packets are swinging. This has been expected since the $\underset{\sim}{\stackrel{+}{\sim}}$ create eigenstates of the Hamiltonian $\underset{\sim}{\sim} \mathrm{HO}$. It also serves as an accuracy test of the integrating routine. The part to the right displays the evolution with interaction at three later times. The occupation probabilities are reshuffled due to the interaction and they fluctuate in time. In fig. 16 (l.h.s.) the chaotic time dependence of $\langle Q(t)|\underset{\sim}{\stackrel{\sim}{\sim}} \underset{\sim}{\sim} \underset{\sim}{\sim}| Q(t)\rangle$ for $n=0,3$ and 6 is depicted.

The result of time averaging is seen in fig. 17 (symbols) for four different initial displacements which correspond to four different excitation energies of the fermion system. To each case we assign a canonical ensemble which has the same mean 

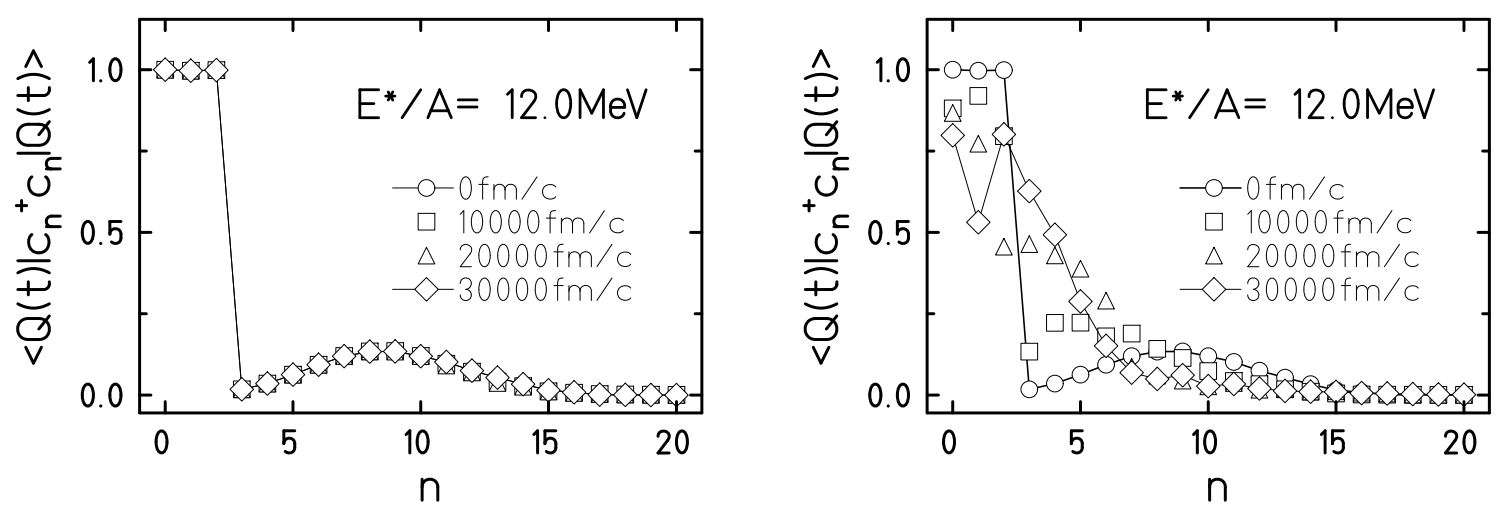

Figure 15: Time evolution of the occupation probabilities for four fermions in a common harmonic oscillator potential without (l.h.s.) and with two-body interaction (r.h.s.). The distributions at $t=0$ and $t=30000 \mathrm{fm} / \mathrm{c}$ are connected by a solid line.
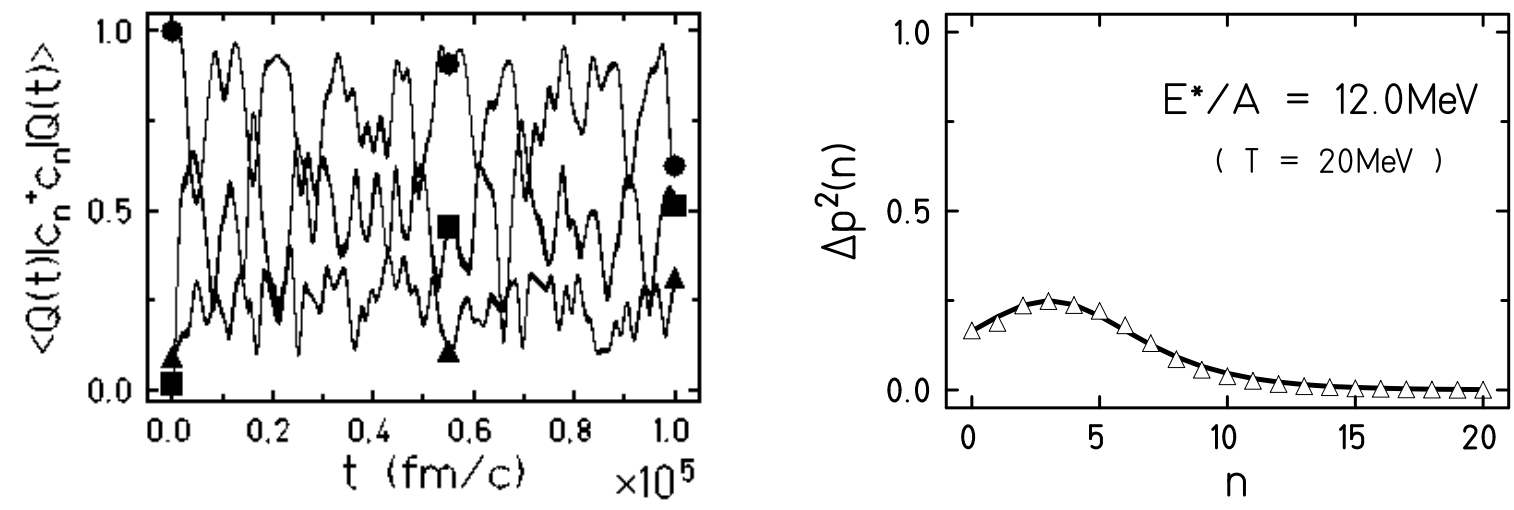

Figure 16: L.h.s.: Occupation probabilities versus time $-n=0$ : circles, $n=3$ : squares, $n=6$ : triangles. R.h.s.: Variance of the fluctuations $\Delta p^{2}(n)$ calculated in the canonical ensemble (solid line) and in the ergodic ensemble (triangles).

energy. The solid lines in fig. 17 show the corresponding distributions of occupation probabilities for these canonical ensembles. Their temperatures $T$ are also quoted in the figure. It is surprising to see that there is almost no difference between the ergodic and the canonical ensemble:

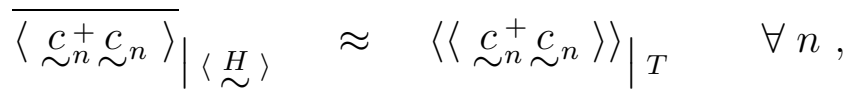




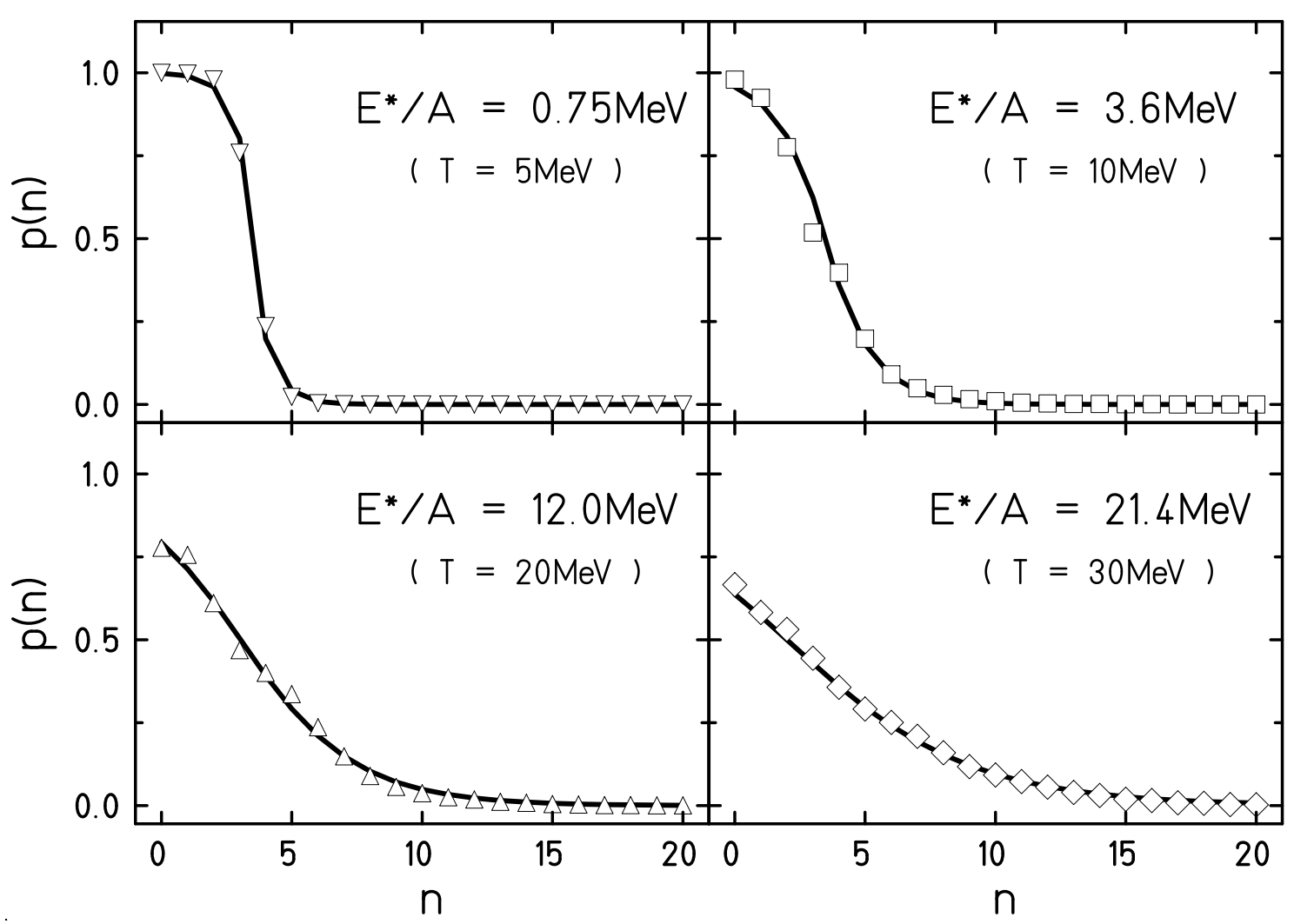

Figure 17: Occupation numbers calculated in the ergodic ensemble (symbols, eq. (72)) compared with the canonical ensemble (solid line, eq. (71)).

provided both have the same excitation energy

$$
E^{*}=\left.\overline{\left\langle{\underset{\sim}{H}}_{\mathrm{HO}}-E_{0}\right\rangle}\right|_{\langle\underset{\sim}{H}\rangle}=\left\langle\left\langle\underset{\sim}{H_{\mathrm{HO}}}-E_{0}\right\rangle\right\rangle_{T_{T}}, \quad E_{0}=8 \omega .
$$

The relation between $E^{*}$ and $T$ is given by eq. (69) and displayed in fig. 14.

This result is not trivial because, firstly, the system is very small, consisting of only four particles, and secondly, the equations of motion are approximated by FMD. The one to one correspondence between the occupation probabilities of the ergodic ensemble and the ones of the canonical ensemble, which has the same mean energy $\langle\underset{\sim}{H}\rangle$ as the pure state, is an impressive demonstration that the system is ergodic and that the FMD many-body trajectory covers the phase space according to Fermi-Dirac statistics.

Not only the one-body distributions of the two ensembles coincide, but also the 
variances of the fluctuations $\Delta p^{2}(n)$,

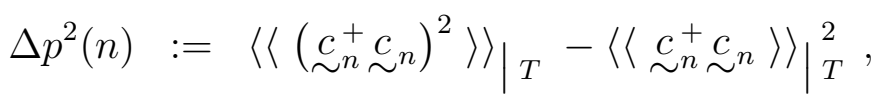

as is demonstrated in fig. 16 (r.h.s.). The ergodic mean converges to the result of the canonical ensemble which is $\Delta p^{2}(n)=p(n)(1-p(n))$.

\subsubsection{Canonical and ergodic ensemble for distinguishable particles}

In this section it is shown that time averaging results in quantum Boltzmann statistics if the fermions are replaced by distinguishable particles. For this end the antisymmetrized many-body state is replaced by a product state of Gaussian wave packets. The resulting equations of motion differ from the FMD case in the skewsymmetric matrix $\mathcal{A}_{\mu \nu}(Q)$ (given in eq. (22) ) which does not couple the generalized velocities of different particles any longer.

For product states the ergodic ensemble is again investigated at different energies and compared with the canonical ensembles with the same mean energies. The appropriate relation between temperatures and excitation energies in the canonical ensemble for distinguishable particles

$$
E^{*}=\left\langle\left\langle\underset{\mathrm{HO}}{H_{\mathrm{HO}}}-E_{0}\right\rangle\right\rangle_{T}=4 \frac{\omega}{2}\left[\operatorname{coth}\left(\frac{\omega}{2 T}\right)-1\right], \quad E_{0}=2 \omega
$$

is shown by the dashed-dotted line in fig. 14.

Since distinguishable particles are not affected by the Pauli principle, the occupation numbers for the many-body ground state look quite different. For instance for zero temperature all particles occupy the eigenstate $|0\rangle$ of the harmonic oscillator (fig. 18, l.h.s.).

The initial single-particle states of the interacting system are chosen analogue to the fermion case. Again the time evolution of the system exhibits ergodic behaviour for all excitation energies. As an example fig. 18 (r.h.s.) is showing the case of $E^{*} / A=2.05 \mathrm{MeV}(T=5 \mathrm{MeV})$ after a time averaging of about 2000 periods. The ergodic ensemble (triangles) and the Boltzmann canonical ensemble (solid line) are the same within the size of the symbols. The result for Fermi-Dirac statistics with the same temperature is included to demonstrate the difference (dotted line). 

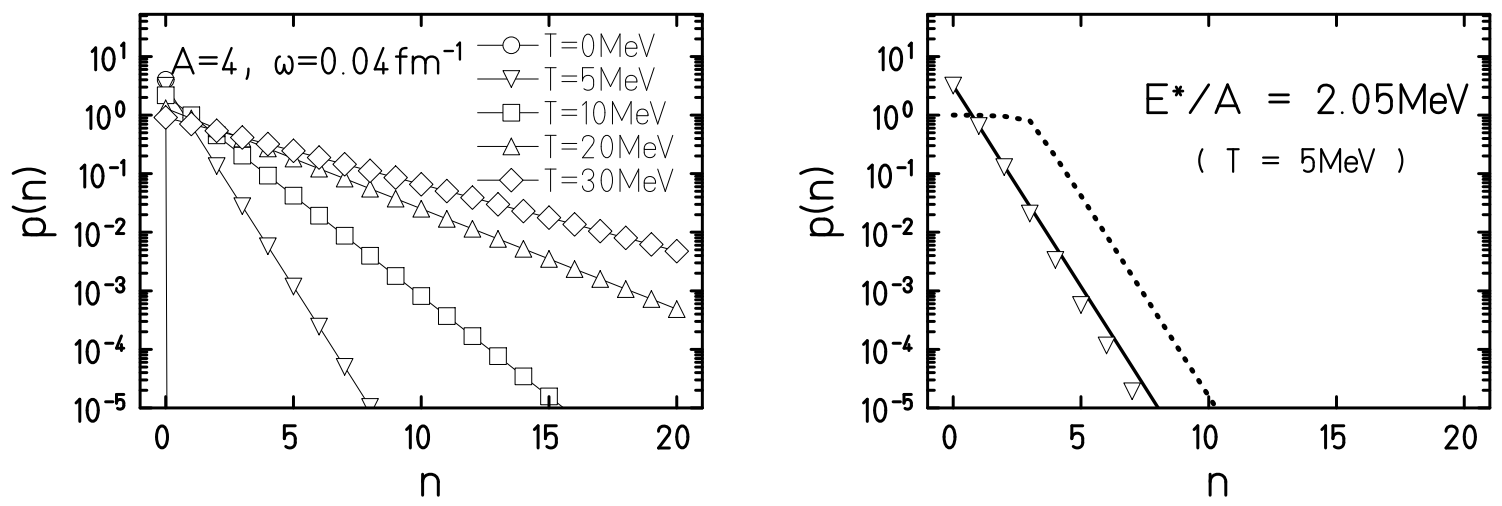

Figure 18: Occupation probabilities for a product state (Boltzmann statistics). L.h.s.: Occupation probabilities $p(n)$ of the oscillator eigenstates for five temperatures for the canonical ensemble. R.h.s: Occupation probabilities calculated in the ergodic ensemble (symbols) compared with those calculated in the canonical ensemble (solid line) for an excitation energy of $E^{*}=2.05 \mathrm{AMeV}$ which corresponds to a temperature $T=5 \mathrm{MeV}$ in the canonical ensemble. The dotted line shows the result for Fermi-Dirac statistics at the same temperature.

This result shows that equations of motion which are not influenced by the Pauli principle lead to the quantum Boltzmann distribution. The only difference to "true classical" equations is the presence of the width parameters as dynamical variables. Only if they are removed from the equations of motion the statistical behaviour of the ergodic ensemble is that of classical statistics.

\subsection{Caloric curve for finite nuclei}

As an outlook of this chapter a method will be presented that allows to investigate the caloric curve of finite charged self-bound Fermi systems like nuclei.

The concept of determining the temperature is to bring a reference system, for which thermodynamic relations between temperature and measurable quantities are known, into thermal equilibrium with the investigated system. The weakly interacting ideal gas, where the temperature is given by the mean kinetic energy of the particles, may serve as an example. The reference system is called a heat bath if its heat capacity is much larger than that of the system and it is called a thermometer 
if its heat capacity is much less.

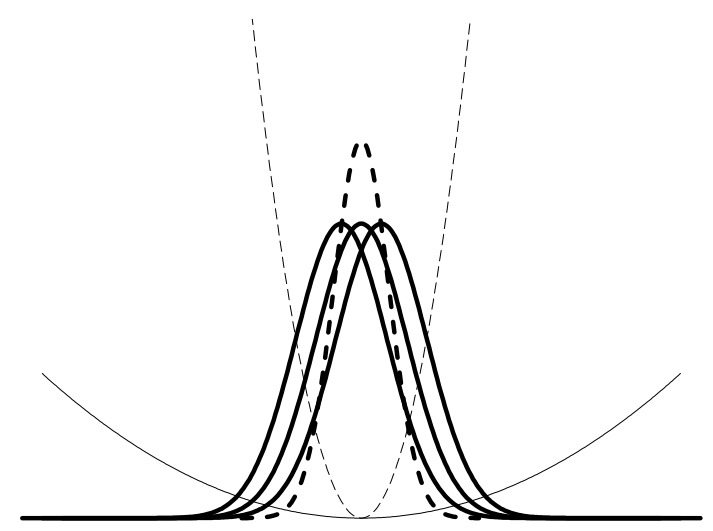

Figure 19: Sketch of the setup: the self-bound excited nuclear system is represented by Gaussian wave packets (solid lines) which are enclosed in a broad container potential. For the thermometer only one wave packet is shown (dashed line), it is contained in a different oscillator.

As the nuclear system is quantal and strongly interacting its temperature cannot be deduced from the momentum distribution or the mean kinetic energy of the nucleons. Therefore, the concept of an external thermometer which is coupled to the nuclear system is used in the present investigation. The thermometer consists of a quantum system of distinguishable particles moving in a common harmonic oscillator potential different from the container potential as shown in fig. 19 .

The time evolution of the whole system is described by the FMD equations of motion. For this purpose the many-body trial state is extended and contains now both, the nucleonic degrees of freedom and the thermometer degrees of freedom

$$
|Q\rangle=\left|Q_{n}\right\rangle \otimes\left|Q_{t h}\right\rangle
$$

The total Hamilton operator including the thermometer is given by

$$
\underset{\sim}{H}=\underset{\sim}{H} n(\omega)+\underset{\sim}{H_{t h}}+\underset{\sim}{H} n-t h
$$

where $\underset{\sim}{H}{ }_{n}(\omega)$ denotes the nuclear Hamiltonian with an additional external field which serves as a container. $\underset{\sim}{H}$ th is the Hamilton operator of the thermometer system, i.e. 
the Hamiltonian of a harmonic oscillator. The week repulsive interaction between thermometer wave packets and nucleons is given by $\underset{\sim}{\sim}$-th .

The determination of the caloric curve is done in the following way. The nucleus is excited by displacing all wave packets from their ground-state positions randomly. Both, centre of mass momentum and angular momentum are kept fixed at zero. To allow a first equilibration between the wave packets of the nucleus and those of the thermometer the system is evolved over a long time $(14000 \mathrm{fm} / \mathrm{c})$. After that a time averaging of the energy of the nucleonic system as well as of the thermometer is performed over 2000 steps covering a time interval of $2000 \mathrm{fm} / \mathrm{c}$. During this time interval the mean of the nucleonic excitation energy

$$
E^{*}=\frac{1}{N_{\text {steps }}} \sum_{i=1}^{N_{\text {steps }}}\left\langle Q_{n}\left(t_{i}\right)\left|\underset{\sim}{H_{n}}\right| Q_{n}\left(t_{i}\right)\right\rangle-E_{0}(N, Z)
$$

is evaluated. $E_{0}(N, Z)$ denotes the FMD ground-state energy of the isotope under consideration. The time-averaged energy of the thermometer $E_{t h}$ which is calculated during the same time interval determines the temperature $T$ through the relation for an ideal gas

$$
T=\omega_{t h}\left[\ln \left(\frac{E_{t h} / N_{t h}+\frac{3}{2} \omega_{t h}}{E_{t h} / N_{t h}-\frac{3}{2} \omega_{t h}}\right)\right]^{-1} .
$$

The system is then cooled and after another $2000 \mathrm{fm} / \mathrm{c}$, in which the system equilibrates, the averaging is done again. Repeating this procedure one follows the caloric curve from high excitations to low excitations.

The relation between the excitation energy and the temperature is evaluated for the three nuclei ${ }^{16} \mathrm{O},{ }^{24} \mathrm{Mg}$ and ${ }^{40} \mathrm{Ca}$ using the same container potential with $\hbar \omega=1 \mathrm{MeV}$.

The caloric curves shown in the graph on the left hand side of fig. 20 clearly exhibit three different parts. Beginning at small excitation energies the temperature rises steeply with increasing energy as expected for the shell model. The nucleons remain bound in the excited nucleus which behaves like a liquid drop of fermions. At an excitation energy of $3 \mathrm{MeV}$ per nucleon the curve flattens and stays almost constant up to about $11 \mathrm{MeV}$. This plateau at $T \approx 5 \mathrm{MeV}$ indicates the coexistence of liquid and vapour phases, the latter consisting of evaporated nucleons which are in equilibrium with the residual liquid drop due to the containment. Around 

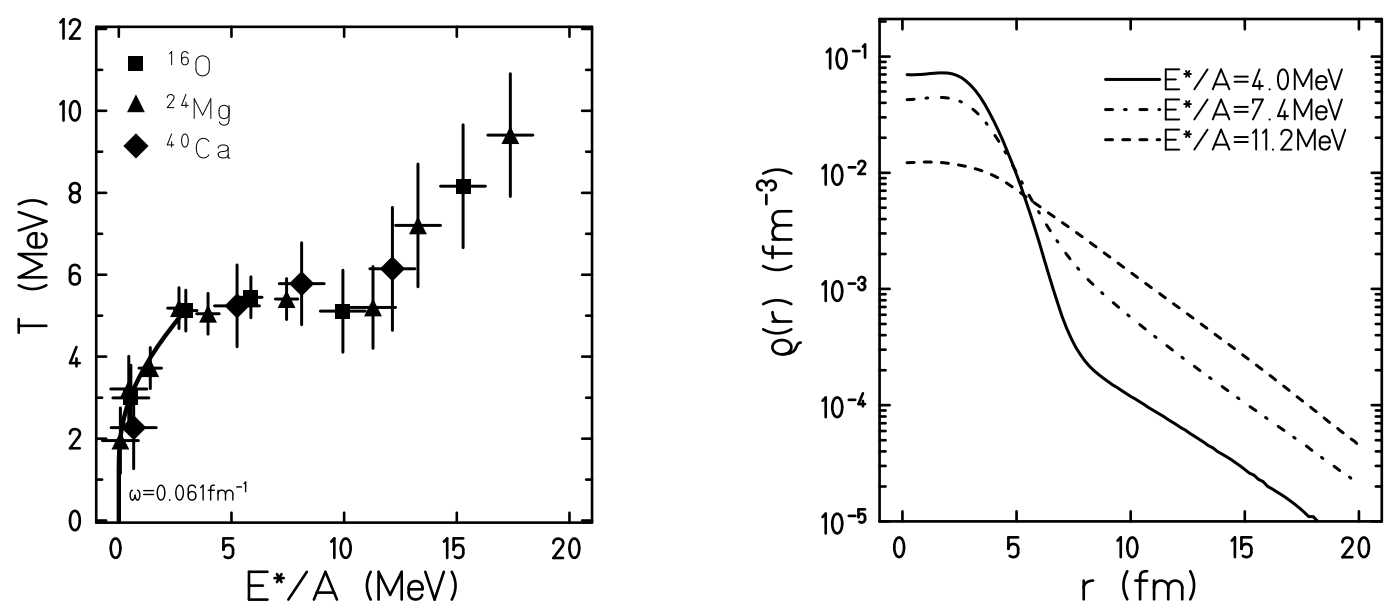

Figure 20: L.h.s.: caloric curve of ${ }^{16} \mathrm{O},{ }^{24} \mathrm{Mg}$ and ${ }^{40} \mathrm{Ca}$ at $\hbar \omega=1 \mathrm{MeV}$, r.h.s.: time averaged radial density distribution of ${ }^{24} \mathrm{Mg}$ at various excitation energies in the coexistence region.

$E^{*} / A \approx 11 \mathrm{MeV}$ all nucleons are unbound and the system has reached the vapour phase. This is indicated by the steep rise of the caloric curve beyond this point. One has to keep in mind that the plateau is not the result of a Maxwell construction as in nuclear matter calculations. In the excitation energy range between 3 and $11 \mathrm{MeV}$ per particle an increasing number of nucleons is found in the vapour phase outside the liquid phase. This can be seen in the density plot on the right hand side of fig. 20, where the radial dependence of the time-averaged density for ${ }^{24} \mathrm{Mg}$ at three excitation energies in the coexistence region is shown. For small excitations $\left(E^{*} / A=4.0 \mathrm{MeV}\right)$ the nucleus, which due to recoil is bouncing around, is surrounded by very low density vapour (solid line). The dashed line $\left(E^{*} / A=7.4 \mathrm{MeV}\right)$ and the dashed-dotted line $\left(E^{*} / A=11.2 \mathrm{MeV}\right)$ show that with increasing energy the vapour contribution is growing and the amount of liquid decreasing. However, in the high energy part of the plateau the averaged one-body density shown here does not represent the physical situation adequately. The time-dependent many-body state shows the formation and disintegration of several small drops, which due to time-averaging cannot be seen in fig. 20. Above $E^{*} / A \approx 13 \mathrm{MeV}$ only vapour is observed.

In how far the experimental result, fig. 21, where the temperature is determined from isotope ratios [56], can be compared to our time-averaged temperature and 


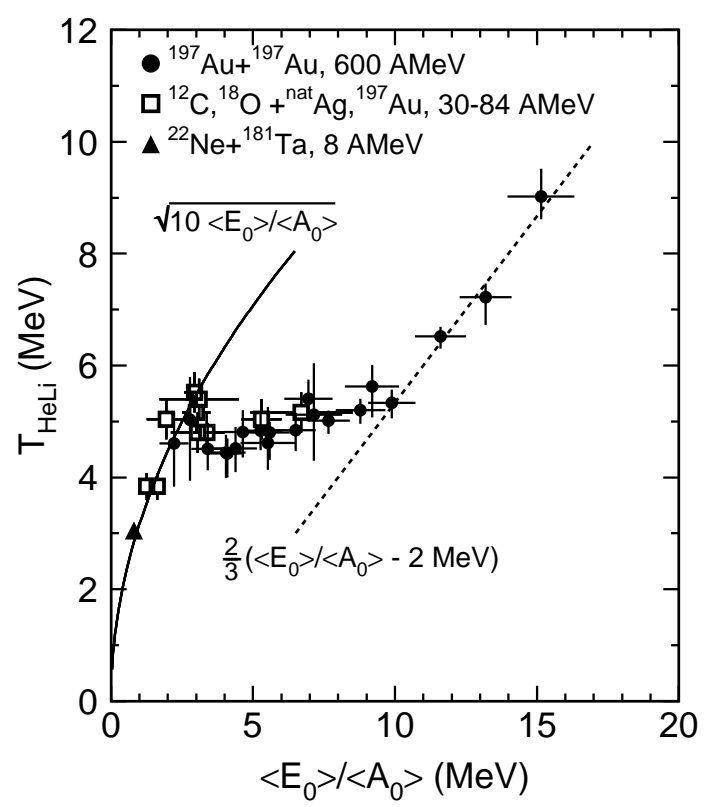

Figure 21: Caloric curve extracted from spectator fragmentation by the ALADIN collaboration (picture taken from ref. [56]).

excitation energy is still under investigation.

The results on the caloric curve of nuclei present another example for the wide applicability of Fermionic Molecular Dynamics. A correct equilibrium behaviour is of course a necessary condition for further investigations of non-equilibrium situations as in nucleus-nucleus collisions. These investigations are followed up presently.

\section{Acknowledgment}

We would like to thank M. Petrovici who kindly provided us with the data on ${ }^{19} \mathrm{~F}-27 \mathrm{Al}$ collisions and J. Pochodzalla who supplied us with the caloric curve of nuclei. We also thank Ph. Chomaz and P. Danielewicz for discussions and T. Neff and R. Roth for carefully reading the manuscript. 


\section{A Interaction matrix elements}

\section{A.1 Approximation of the matrix elements}

A large fraction of the numerical effort of FMD is caused by the evaluation of the interaction matrix elements

$$
\mathcal{V}(Q) \equiv\langle\underset{\sim}{V}\rangle=\operatorname{Tr}\left(\underset{\sim}{\sim} \rho^{(2)}\right)=\frac{1}{2} \sum_{k, l, m, n}^{A}\left\langle q_{k} q_{l}|\underset{\sim}{\sim}| q_{m} q_{n}\right\rangle\left(\mathcal{O}_{m k} \mathcal{O}_{n l}-\mathcal{O}_{m l} \mathcal{O}_{n k}\right)
$$

This effort grows with $A^{4}$ because Gaussian wave packets are not orthogonal. In order to reduce the computation time two types of approximations were tested [45, 46]. Both approximation schemes use that antisymmetrization effectively reduces the strength of the interaction.

The first ansatz [45]

$$
\begin{aligned}
\mathcal{V}(Q) \approx \sum_{k<l} \frac{\left\langle q_{k} q_{l}|\underset{\sim}{\sim}| q_{k} q_{l}\right\rangle_{a}}{\left\langle q_{k} q_{l} \mid q_{k} q_{l}\right\rangle} \exp \left\{-\frac{1}{4} c_{k l}\right\} \\
c_{k l}=\sum_{m \neq k, l}^{A}\left(\frac{\left\langle q_{k} q_{m} \mid q_{m} q_{k}\right\rangle}{\left\langle q_{k} q_{m} \mid q_{k} q_{m}\right\rangle}+\frac{\left\langle q_{l} q_{m} \mid q_{m} q_{l}\right\rangle}{\left\langle q_{l} q_{m} \mid q_{l} q_{m}\right\rangle}\right) .
\end{aligned}
$$

takes all matrix elements $\left\langle q_{k} q_{l}|\underset{\sim}{\sim}| q_{k} q_{l}\right\rangle$ and $\left\langle q_{k} q_{l}|\underset{\sim}{\sim}| q_{l} q_{k}\right\rangle$ into account which are scaled with an overall factor counting the overlapping wave packets.

The second ansatz [46] reduces this effort even further since it needs only the direct matrix elements

$$
\begin{aligned}
\mathcal{V}(Q) & \approx \sum_{k<l} \frac{\left\langle q_{k} q_{l}|\underset{\sim}{\operatorname{V}}| q_{k} q_{l}\right\rangle_{a}}{\left\langle q_{k} q_{l} \mid q_{k} q_{l}\right\rangle}\left(\left(1-\gamma_{k l}\right)+\gamma_{k l} \exp \left\{-\frac{\left\langle q_{k} q_{l} \mid q_{l} q_{k}\right\rangle}{\left\langle q_{k} q_{l} \mid q_{k} q_{l}\right\rangle}\right\}\right) \\
\gamma_{k l} & =\operatorname{erf}\left\{\frac{1}{2}+\sum_{m \neq k, l}^{A}\left(\frac{\left\langle q_{k} q_{m} \mid q_{m} q_{k}\right\rangle}{\left\langle q_{k} q_{m} \mid q_{k} q_{m}\right\rangle}+\frac{\left\langle q_{l} q_{m} \mid q_{m} q_{l}\right\rangle}{\left\langle q_{l} q_{m} \mid q_{l} q_{m}\right\rangle}\right)\right\} .
\end{aligned}
$$

Both schemes (82) and (83) provide a reasonable approximation for the expectation value of the two-body interaction. In order to reproduce the nuclear binding energies it can be necessary to readjust the strength of the original interaction. 


\section{A.2 Coulomb interaction}

This section briefly shows how the second approximation (83) works for the Coulomb interaction (40)

$$
\left\langle\vec{x}_{i}, \vec{x}_{j}\left|{\underset{\sim}{\sim}}_{c}(i, j)\right| \vec{x}_{k}, \vec{x}_{l}\right\rangle=\frac{1.44 \mathrm{MeVfm}}{\left|\vec{x}_{i}-\vec{x}_{j}\right|} \underset{\sim}{\sim} \otimes \underset{\sim}{\sim} \stackrel{P}{p}^{3}\left(\vec{x}_{i}-\vec{x}_{k}\right) \delta^{3}\left(\vec{x}_{j}-\vec{x}_{l}\right)
$$

where $\underset{\sim}{P}$ denotes the projection operator on the protons. The diagonal matrix elements are

$$
\frac{\left\langle q_{k} q_{l}|\underset{\sim}{\sim}| q_{k} q_{l}\right\rangle}{\left\langle q_{k} q_{l} \mid q_{k} q_{l}\right\rangle}=\frac{1.44 \mathrm{MeVfm}}{r_{k l}} \operatorname{erf}\left\{\sqrt{\frac{\lambda_{k l k l}}{2}} r_{k l}\right\} P_{k k}^{p} P_{l l}^{p}
$$

with

$$
\begin{aligned}
r_{k l} & =\left|\vec{r}_{k}-\vec{r}_{l}\right| \\
\lambda_{k l k l} & =\frac{2 a_{k R} a_{l R}}{a_{k R}\left|a_{l}\right|^{2}+a_{l R}\left|a_{k}\right|^{2}} \\
\operatorname{erf}(x) & :=\frac{2}{\sqrt{\pi}} \int_{0}^{x} d u \exp \left\{-u^{2}\right\} \\
P_{k l}^{p} & =\left\langle\xi_{k}\left|\stackrel{P}{\sim}^{p}\right| \xi_{l}\right\rangle=\frac{1}{2}\left(1+\xi_{l}\right)\left\langle\xi_{k} \mid \xi_{l}\right\rangle
\end{aligned}
$$

The isospin variable $\xi_{k}$ takes values $\xi_{k}=1$ for protons and $\xi_{k}=-1$ for neutrons, respectively.

Figure 22 shows the result of the approximation for two systems, two identical protons and a ${ }^{12} \mathrm{C}$ nucleus. The approximation (thin line) compares nicely to the exact result (thick line). 

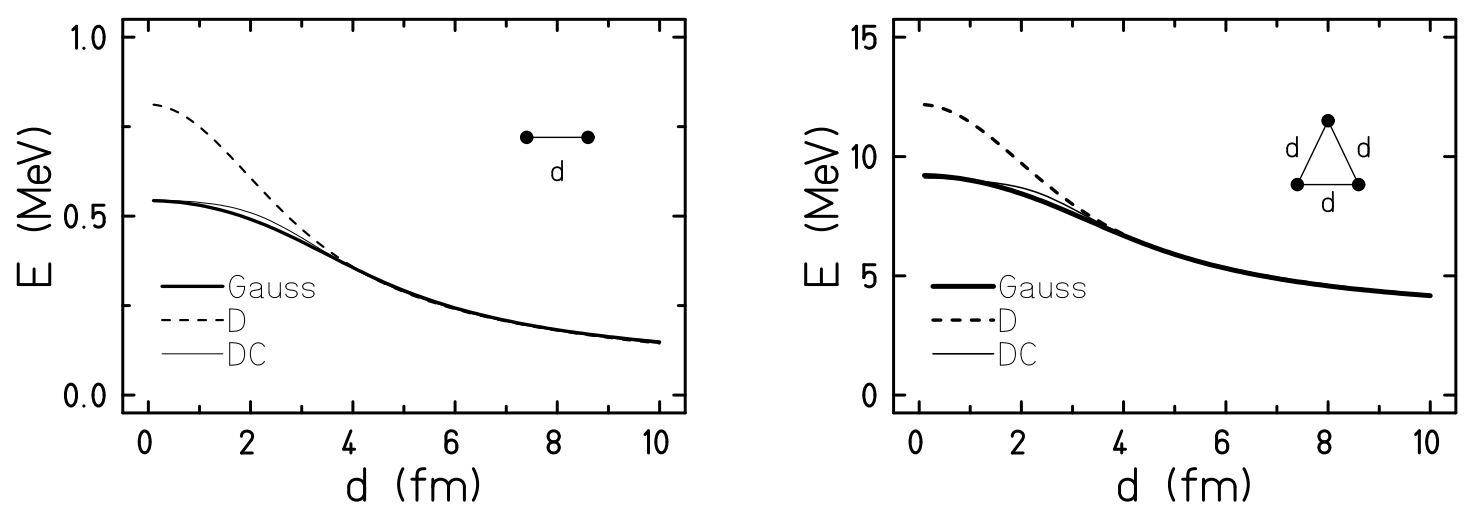

Figure 22: Approximation of the Coulomb energy. The 1.h.s shows the Coulomb energy of two protons with the same spin component as a function of the relative distance, the r.h.s. shows the Coulomb energy of a ${ }^{12} \mathrm{C}$ nucleus as a function of the relative distance between the three alpha clusters. The thick solid line represents the exact solution, the dashed line the direct term only and the thin line the result of the approximation.

\section{References}

[1] W.U. Schröder, J.R. Huizenga, in Treatise on Heavy Ion Science, ed. D.A. Bromley, vol. 2, p. 115, Plenum Press New York and London (1984)

[2] A. Gobbi, W. Nörenberg, in Heavy Ion Collisions, ed. R. Bock, vol. 2, p. 127, North Holland Publ. Company (1980)

[3] J. Blocki, Y. Bonch, J.R. Nix, J. Randrup, M. Nobel, A.J. Sierk, W.J. Swiatecki, Ann. Phys. (NY) 113 (1978) 330

[4] J. Randrup, Nucl. Phys. A327 (1979) 490 and Nucl. Phys. A383 (1982) 468

[5] H. Feldmeier, Rep. Prog. Phys. 50 (1987) 915

[6] K.T.R. Davies, K.R.S. Devi, S.E. Koonin, M.R. Strayer, in Treatise on Heavy Ion Science, ed. D.A. Bromley, vol. 3, p. 1, Plenum Press New York and London (1985)

[7] J.A. Maruhn, R.Y. Cusson, Nucl. Phys. A270 (1976) 471 
[8] S.E. Koonin, K.T.R. Davies, V. Maruhn-Rezwani, H.T. Feldmeier, S.J. Krieger, J.W. Negele, Phys. Rev. C15 (1977) 1359

[9] K.T.R. Davies, H.T. Feldmeier, H. Flocard, M.S. Weiss, Phys. Rev. C18 (1978) 2631

[10] J.J. Griffin, P.C. Lichtner, M. Dworzecka, Phys. Rev. C21 (1980) 1351

[11] G.F. Bertsch, H. Kruse, S. Das Gupta, Phys. Rev. C29 (1984) 673

[12] H. Kruse, B. Jacak, H. Stöcker, Phys. Rev. Lett. 54 (1985) 289

[13] W. Bauer, G.F. Bertsch, W. Cassing, U. Mosel, Phys. Rev. C34 (1986) 2127

[14] C. Grégoire, B. Remaud, F. Sébille, L. Vinet, Y. Raffray, Nucl. Phys. A465 (1987) 317

[15] G.F. Bertsch, S. Das Gupta, Phys. Rep. 160 (1988) 189

[16] A. Bonasera, M. Di Toro, H.H. Wolter, Proc. XXVII Winter Meeting, eds. I. Iori et al., Bormio (1989)

[17] J. Aichelin, H. Stöcker, Phys. Lett. B176 (1986) 14

[18] J. Aichelin, Phys. Rep. 202 (1991) 233

[19] J. Jaenicke, J. Aichelin, N. Ohtsuka, R. Lineden, A. Faessler, Nucl. Phys. A536 (1992) 102

[20] D.T. Khoa, N. Ohtsuka, M.A. Matin, A. Faessler, S.W. Huang, E. Lehmann, R.K. Puri, Nucl. Phys. A548 (1992) 102

[21] L.Wilets, E.M.Henley, M.Kraft, A.D.MacKellar, Nucl.Phys. A282 (1977) 341

[22] D.H. Boal, J.N. Glosli, Phys. Rev. C38 (1988) 1870

[23] D.H.Boal, J.N.Glosli, Phys. Rev. C38 (1988) 2621

[24] C. Dorso, S. Duarte, J. Randrup, Phys. Lett. B188 (1987) 287

[25] C. Dorso, J. Randrup, Phys.Lett. B215 (1987) 611

[26] C. Dorso, J. Randrup, Phys.Lett. B232 (1989) 29 
[27] H. Feldmeier, Nucl. Phys. A515 (1990) 147

[28] H. Feldmeier, K. Bieler, J. Schnack, Nucl. Phys. A586 (1995) 493

[29] J.Schnack, H. Feldmeier, Nucl. Phys. A601 (1996) 181

[30] P. Kramer, M. Saraceno, Lecture Notes in Physics 140, Springer, Berlin (1981)

[31] V. Bargmann, L. Michel, V. Telegdi, Phys. Rev. Letters 2 (1959) 435

[32] C. Coriano, R. Parwani, H. Yamagishi, Nucl. Phys. A522 (1991) 591

[33] V.I. Arnol'd, Mathematical Methods of Classical Mechanics, Springer-Verlag Berlin (1989)

[34] J. Broeckhove, L. Lathouwers, P. van Leuven, J. Phys. A: Math. Gen. 22 (1989) 4395

[35] D. Kiederlen, P. Danielewicz, preprint, MSUCL-1047 (1996)

[36] K. Yoshihara, H. Kudo, T. Sekine, Periodic Table with Nuclides and Reference Data, Springer-Verlag, Berlin (1985)

[37] P. Møller, J.R. Nix, W.D. Myers, W.J. Swiatecki, Atomic Data Nucl. Data Tables 59, (1995) 185

[38] Landolt-Börnstein, Zahlenwerte und Funktionen aus Naturwissenschaften und Technik, Gruppe I, Band 2: Kernradien, Springer-Verlag, Berlin (1967)

[39] T. Angeli, Acta Physica Hungarica 69 (1991) 233

[40] G. Peilert, J. Konopka, H. Stöcker, W. Greiner, M. Blann, M.G. Mustafa, Phys. Rev. C46 (1992) 1457

[41] S.A. Bass, C. Hartnack, H. Stöcker, W. Greiner, Phys. Rev. C51 (1995) 3343

[42] J. Konopka, dissertation, Johann Wolfgang Goethe-Universität, Frankfurt am Main (1996)

[43] T. Maruyama, K. Niita, A. Iwamoto, Phys. Rev. C53 (1996) 297

[44] H. Feldmeier, J. Schnack, Proc. Int. Workshop on Dyn. Features of Nuclei, Sitges, Spain (1993) World Scientific 
[45] J. Schnack, diploma thesis, GSI report GSI-93-21 (1993)

[46] J. Schnack, dissertation, TH Darmstadt (1996) (copy from http://www.gsi.de/ schnack)

[47] J. Knoll, B. Strack, Phys. Lett. B149 (1984) 45

[48] J. Knoll, J. Wu, Nucl. Phys. A481 (1988) 173

[49] H. Feldmeier, J. Schnack, Nucl. Phys. A583 (1995) 347c

[50] M. Petrovici, A. Adronic, I. Berceanu, A. Buţă, M. Duma, D.Moisă, A. Pop, V. Simion, A. Bonasera, G. Immé, G. Lanzanò, A. Pagano, G. Raciti, N. Colonna, G. d'Erasmo, A. Pantaleo, Z. Phys. A354 (1996) 11;

A. Pop et al. Proc. of the INPC, Beijing 1995, p. 5.2-2

[51] H.J. Krappe, J.R. Nix, A.J. Sierk, Phys. Rev. C20 (1979) 992

[52] Multifragmentation,Proceedings of the International Workshop XXII on Gross Properties of Nuclei and Nuclear Excitations, eds. H. Feldmeier \& W. Nörenberg, Hirschegg, Austria (1994)

[53] J.P. Bondorf, A.S. Botvina, A.S. Ilinov, I.N. Mishustin, K. Sneppen, Phys. Rep. 257 (1995) 133

[54] D.H.E. Gross, Rep. Prog. Phys. 53 (1990) 605

[55] W.A. Friedman, Phys. Rev. Letts. 60 (1988) 2125;

W.A. Friedman, Phys. Rev. C42 (1990) 667

[56] J. Pochodzalla, T. Möhlenkamp, T. Rubehn, A. Schüttauf, A. Wörner, E. Zude, M. Begemann-Blaich, Th. Blaich, C. Gross, H. Emling, A. Ferrero, G. Imme, I. Iori, G.J. Kunde, W.D. Kunze, V. Lindenstruth, U. Lynen, A. Morini, W.F.J. Müller, B. Ocker, G. Raciti, H. Sann, C. Schwarz, W. Seidel, V. Serfling, J. Stroth, A. Trzcinski, W. Trautmann, A. Tucholski, G. Verde, B. Zwieglinski, Phys. Rev. Lett. 75 (1995) 1040

[57] J.B. Natowitz, K. Hagel, R. Wada, Z. Majka, P. Gonthier, J. Li, N. Mdeiwayeh, B. Xiao, Y. Zhao, Phys. Rev. C52 (1995) R2322 
[58] W. Reisdorf et al.(FOPI collaboration), in press, Nucl. Phys. A (1996); copy from nucl-ex/9610009

[59] K. Hagel, M. Gonin, R. Wada, J.B. Natowitz, F. Haddad, Y. Lou, M. Gui, D. Utley, B. Xiaó, J. Li, G. Nebbia, D. Fabris, G. Prete, J. Ruiz, D. Drain, B. Chombon, B. Cheynis, D. Guinet, X.C. Hu, A. Demeyer, C. Pastor, A. Giorni, A. Lleres, P. Stassi, J.B. Viano, P. Gonthier, Phys. Rev. C50 (1994) 2017

[60] Ph. Chomaz, M. Colonna, A. Guarnera, Proc. 12th Winter Workshop on Nuclear Dynamics, Snowbird, ed. W. Bauer, Plenum, New York (1996) 65

[61] A. Ono, H. Horiuchi, Toshiki Maruyama, A. Ohnishi, Phys. Rev. Lett. 68 (1992) 2898; Phys. Rev. C47 (1993) 2652

[62] A. Ono, H. Horiuchi, Toshiki Maruyama, Phys. Rev. C48 (1993) 2946; A. Ono, H. Horiuchi, Phys. Rev. C51 (1995) 299; E.I. Tanaka, A. Ono, H. Horiuchi, Tomoyuki Maruyama, A. Engel, Phys. Rev. C52 (1995) 316

[63] A. Ono, H. Horiuchi, Phys. Rev. C53 (1996) 2958

[64] A. Ohnishi, J. Randrup, Nucl. Phys. A565 (1993) 474

[65] A. Ohnishi, J. Randrup, Phys. Rev. Lett. 75 (1995) 596

[66] L.G. Moretto et al., Phys. Rev. Lett. 76 (1996) 2822;

J. Pochodzalla et al., Phys. Rev. Lett. 76 (1996) 2823

[67] H.R. Jaqaman, A.Z. Mekjian, L. Zamik, Phys. Rev. C 29 (1984) 2067

[68] A.L. Goodman, J.I. Kapusta, A.Z. Mekjian, Phys. Rev. C 30 (1984) 851

[69] P. Bonche, S. Levit, D. Vautherin, Nucl. Phys. A427 (1984) 278-296

[70] P. Bonche, S. Levit, D. Vautherin, Nucl. Phys. A436 (1985) 265-293

[71] S. Levit, P. Bonche, Nucl. Phys. A437 (1985) 426

[72] G. Papp, W. Nörenberg, APH Heavy Ion Physics 1 (1995) 241

[73] J.Schnack, H. Feldmeier, GSI-Preprint-97-18 
[74] J.R. Klauder, B.-S. Skagerstam, Coherent States, World Scientific Publishing Co. Pte. Ltd., Singapore (1985) 


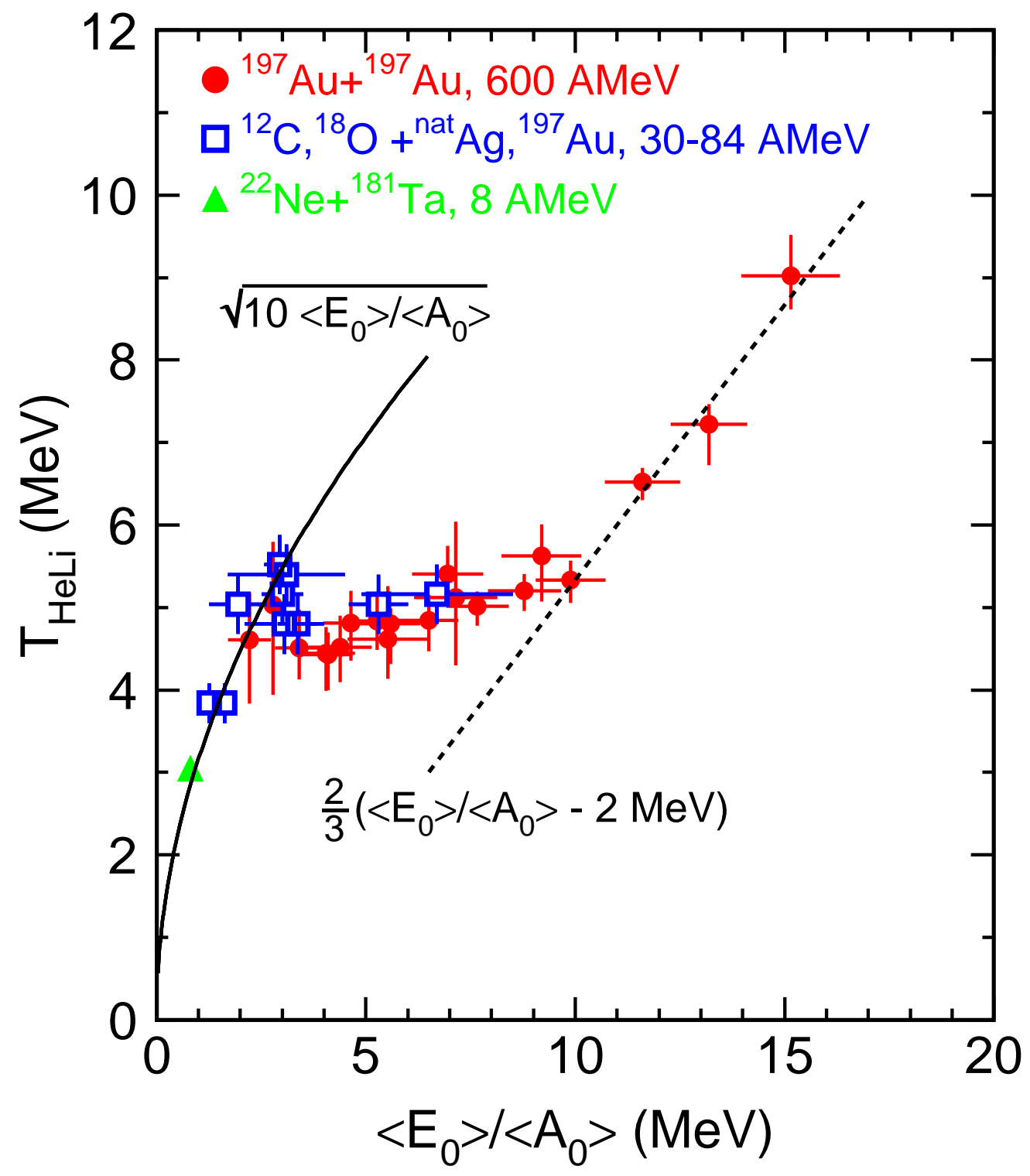




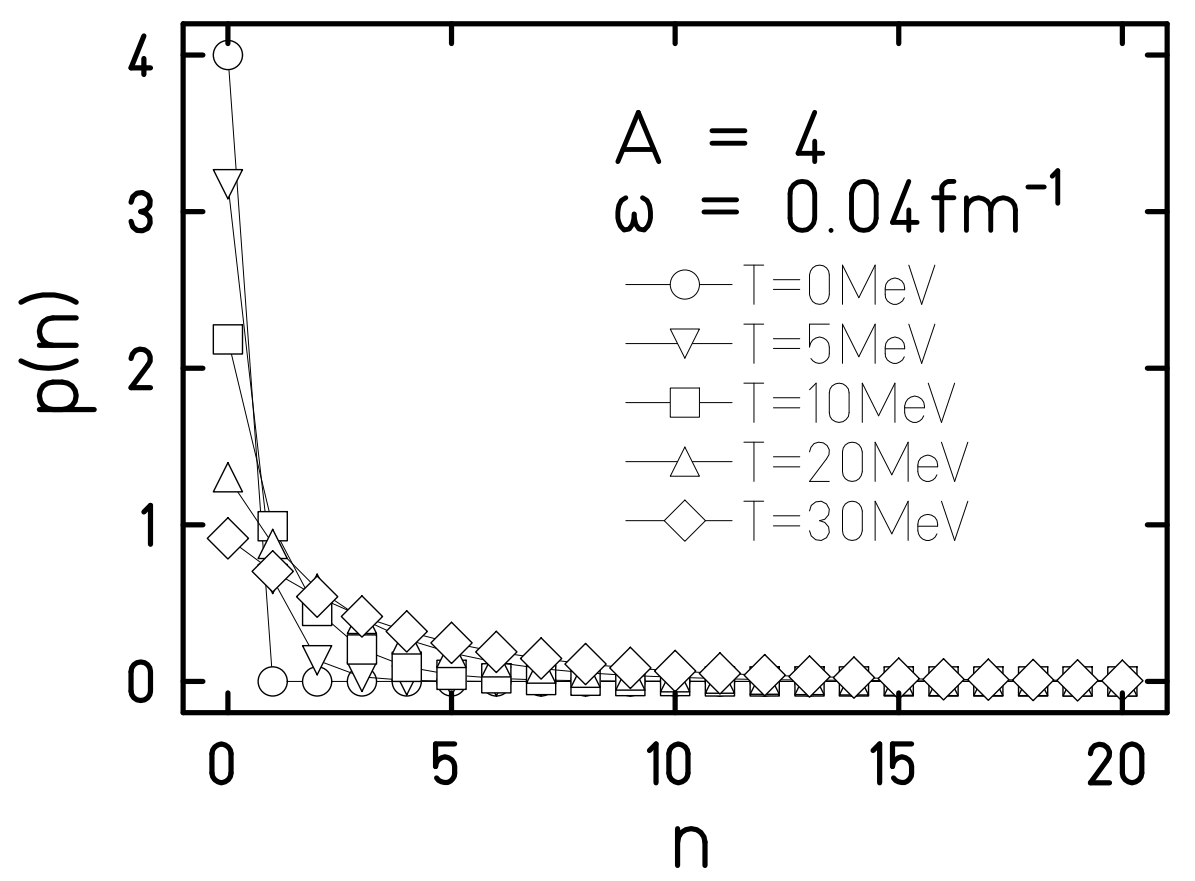




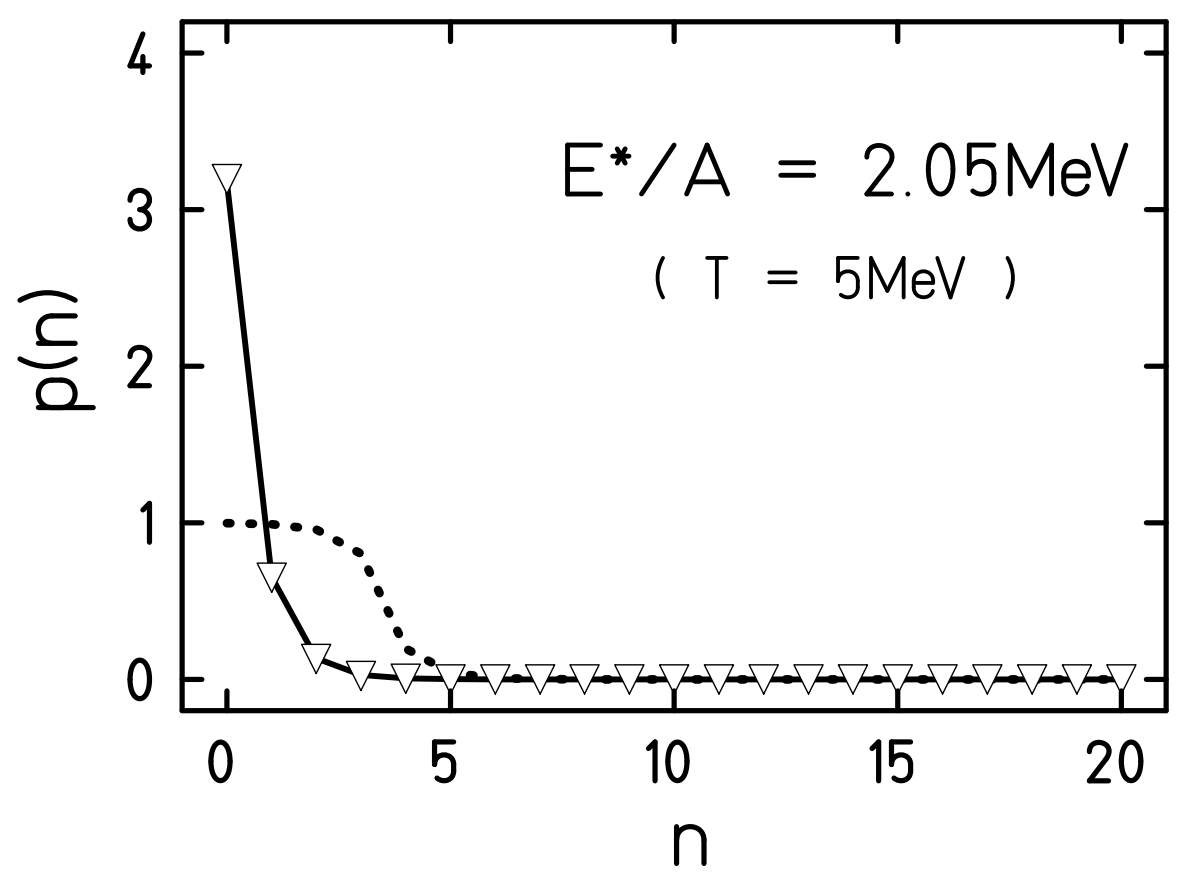

